\title{
ON THE LEGACY OF FREE DIVISORS III: FUNCTIONS AND DIVISORS ON COMPLETE INTERSECTIONS
}

\author{
JAMES DAMON \\ Department of Mathematics \\ University of North Carolina at Chapel Hill, \\ Chapel Hill, NC 27599-3250, USA
}

\section{INTRODUCTION}

Many of the key properties of (complex) isolated complete intersection singularities are encoded in the discriminants of their versal unfoldings. This includes the Milnor number, monodromy, and deformation properties. An especially important property of discriminants and bifurcation sets which has important consequences for their topology is that they are free divisors, e.g. by the work of Arnold [A1], [A2], Saito [Sa], Looijenga [L], Bruce [Br], Terao [To], etc. Most of these results establishing freeness for both discriminants and bifurcation sets follow from a more general result concerning the freeness of discriminants for $\mathcal{K}_{V}$ equivalence, which captures the equivalence of germs of varieties obtained as nonlinear sections of $V$ [D5], [D6]. Furthermore, David Mond and this author [DM], [D2], [D5], [Mo] have shown the fundamental role of freeness for determining the topology of discriminants of mappings, and more generally nonisolated singularities arising as nonlinear sections of free divisors.

This is just one example illustrating the importance of understanding properties of singularities in the relative case where properties of mappings, singularities, and divisors are determined relative to a subvariety $V$ in source or target. Instead the equivalence can preserve a fixed hypersurface $V$ in the source. Then, $V$ can be viewed as a "boundary" and the equivalence becomes that for "boundary singularities"investigated by Siersma [Si], Arnold [A1], [A2], Lyashko [Ly], Bruce and Giblin [BG1] [BG2], Tari [Ta], etc. A second case concerns equivalence of germs of mappings, divisors, or subvarieties on a singular variety $V$, where either the variety $V$ is fixed (which goes back to Galligo's work on stability [Ga]) or is also allowed to vary.

In several special cases, Arnold, Lyashko, and Zakalyukin have shown the freeness of bifurcation sets of "simple boundary singularities". Also, results of Goryunov [Go2] combined with Mond and Van Straten [MVS] established the freeness of the bifurcation set for functions on space curves in $\mathbb{C}^{3}$. These special results suggest more general results might be true for equivalences preserving varieties. In this paper, we shall apply results from parts I and II [D5] and [D6], to identify when discriminants and bifurcation sets are free for the above types of equivalences for the case of holomorphic germs and germs of complex analytic varieties $V$.

Partially supported by National Science Foundation grants DMS-0103862 and DMS-0405947. 
Equivalences for maps $\mathbb{C}^{n}, 0 \rightarrow \mathbb{C}^{p}, 0$ relative to variety $V, 0$.

Relative Equivalence Groups $\quad \begin{aligned} & \text { Bifurcation Sets, } \\ & \text { Discriminants }\end{aligned}$

i) Fixed hypersurface $V, 0 \subset \mathbb{C}^{n}, 0 \quad{ }_{V}^{\mathcal{K}}, \quad$ discriminants equivalence preserving $V \quad{ }_{V} \mathcal{A},{ }_{V} \mathcal{R}^{+} \quad$ bifurcation sets

ii) Fixed hypersurface $V, 0 \subset \mathbb{C}^{p}, 0 \quad \mathcal{K}_{V} \quad$ discriminants $\mathcal{K}$-equivalence preserving $V$

iii) Fixed complete Intersection $V, 0 \subset \mathbb{C}^{n}, 0$, and equivalence for map germs on $V$

iv) Varying hypersurface $X_{0}, 0$ on $\quad \mathcal{K}_{V_{2}, V_{1}}$ discriminants varying complete intersection $X, 0 \subset \mathbb{C}^{n}, 0$

v) Varying hypersurface $X_{0}, 0$ on $\quad \mathcal{K}_{\mathbb{C}^{p}, V_{1}} \quad$ discriminants varying ICIS $X, 0 \subset \mathbb{C}^{n}, 0$

Table 1: Relative Equivalences and Their Associated Geometric Subgroups

We consider modifications of the equivalences $\mathcal{A}, \mathcal{K}$, and $\mathcal{R}$ to preserve $V$, a nonisolated complete intersection or hypersurface singularity. These will include: 1) the equivalence of functions and mappings preserving a free divisor in the source (i.e. "boundary singularities") as i) in Table 1;2) divisors, functions, or map germs on an "almost free" complete intersection where both are allowed to vary, as iv) and $\mathrm{v}$ ) in Table 1; and 3) complete intersection map germs on fixed free complete intersections as iii) in Table 1.

These equivalence groups are "geometric subgroups" $\mathcal{G}$ of $\mathcal{A}$ or $\mathcal{K}$ (see [D3] and $[\mathrm{D} 4, \S 8])$. Hence, germs which have finite codimension for the equivalence $\mathcal{G}$ have $\mathcal{G}$-versal unfoldings (and there is a criteria for $\mathcal{G}$-stability of germs). We shall apply the general criteria given in part II [D6] to determine when the discriminants for $\mathcal{G}$-stable germs or bifurcation sets for $\mathcal{G}$-versal unfoldings are free divisors.

Freeness is explained in Part II by the motto

\section{Cohen-Macaulay of codim $1+$ Genericity of Morse Type Singularities \\ $\Longrightarrow$ Freeness of Discriminants}

Here "discriminants" are understood in a generalized sense which includes both discriminants and bifurcation sets (we shall use the term "discriminant" except when we distinguish "bifurcation sets" for $\mathcal{A}$-type equivalences from discriminants of $\mathcal{A}-$ type stable germs).

We remark that Morse-type singularities are quite different from usual Morse singularities. They differ for each equivalence group $\mathcal{G}$, and may only be generic in a restricted dimension range. Morse-type singularities for $\mathcal{K}_{V}$-equivalence then play a special role. They contain topological information about $V$ exhibited by sections of varying dimensions. In addition, despite the considerable differences 
among the Morse-type singularities for various equivalence relations we consider, we shall classify them in terms of Morse-type singularities for $\mathcal{K}_{V}$-equivalence. Consequently, throughout this paper, we shall refer to "Morse-type singularities of $V$ ", which will always mean for $\mathcal{K}_{V}$-equivalence.

Second, "Cohen-Macaulay of codim 1"is realized when the geometric subgroup $\mathcal{G}$ is "Cohen-Macaulay" in the sense of Part II. If $\mathcal{G}$ is Cohen-Macaulay, without the genericity of Morse- type singularities, or $\mathcal{G}$ is not Cohen-Macaulay, but has a "Cohen-Macaulay Reduction" (CM-reduction) $\mathcal{G}^{*}$ in the sense of Part II, then the $\mathcal{G}$-discriminant will still have a weaker free* divisor structure defined by the module of $\mathcal{G}$-liftable, resp. $\mathcal{G}^{*}$-liftable, vector fields. This still allows us to determine the (vanishing) topology of the discriminant (or bifurcation set).

For $\mathcal{K}_{V}$-equivalence of germs viewed as sections of a free divisor $V$, the criterion for the freeness of $\mathcal{K}_{V}$-discriminants reduces to the conditions given in part I. We include $\mathcal{K}_{V}$-equivalence here because it plays an important role in understanding the conditions for the other equivalences.

First, in $\S 2$ we consider equivalences of germs $f_{0}: \mathbb{C}^{n} \rightarrow \mathbb{C}^{p}, 0$ which preserve a fixed free divisor in the source $V, 0 \subset \mathbb{C}^{n}, 0$ (denoting the groups ${ }_{V} \mathcal{K},{ }_{V} \mathcal{A}$, and $\left.{ }_{V} \mathcal{R}^{+}\right)$. In Theorem 1 we first prove that ${ }_{V} \mathcal{K}$ is Cohen-Macaulay provided $n-p<$ $h n(V)$, the holonomic codimension of $V$. Hence, the ${ }_{V} \mathcal{K}$-discriminant has a free* divisor structure defined by the module of ${ }_{V} \mathcal{K}$-liftable vector fields. If in addition, $V, 0$ generically has Morse-type singularities in dimension $n-p$, then the ${ }_{V} \mathcal{K}-$ discriminant is a free divisor. We prove this by establishing in $\S 6$ a natural "dual correspondence" between Morse-type singularities for $\mathcal{K}_{V}$ and ${ }_{V} \mathcal{K}$-equivalences.

We then apply this result to deduce corresponding results for the freeness (or obtaining free* divisor structures) for ${ }_{V} \mathcal{A}$-discriminants for ${ }_{V} \mathcal{A}$-stable germs (Theorem 2) and for ${ }_{V} \mathcal{R}^{+}$-discriminants for ${ }_{V} \mathcal{R}^{+}$-versal unfoldings (Corollary 3 ). Lastly, in Theorem 4, we prove the ${ }_{V} \mathcal{A}$-bifurcation set is a free divisor for germs belonging to a ${ }_{V} \mathcal{A}$-distinguished bifurcation class (an analogue of Theorem 3 of part I for $\mathcal{A}$-bifurcation sets).

These theorems are applied in $\S 2$ to all of the "boundary singularities" mentioned earlier. One unexpected result is that although Arnold and Lyashko showed for simple boundary singularities of functions that the discriminants for versal unfoldings are free divisors, this does not hold in general for nonsimple boundary singularities. Whether it holds depends upon whether the singular boundary $V$ generically has Morse-type singularities. Then, if $V$ is a free divisor and generically has Morsetype singularities in dimension $n-p$, then freeness of ${ }_{V} \mathcal{K}$-discriminants also holds for mappings $\mathbb{C}^{n}, 0 \rightarrow \mathbb{C}^{p}, 0$.

Second, in $\S 3$ we consider the relative situation of a divisor $X_{0}$ on a (nonisolated) complete intersection $X$, where we allow both to vary. The complete intersection is allowed to deform as an "almost free complete intersection", which is a nonlinear section of a free complete intersection (ICIS singularities are the simplest examples). For this situation,we consider a relative form of $\mathcal{K}_{V}$-equivalence, denoted $\mathcal{K}_{V_{2}, V_{1}}$, for a nonlinear section $f_{0}$ of a pair $V_{1}, 0 \subset V_{2}, 0$ so $\left(X, X_{0}\right)=\left(f_{0}^{-1}\left(V_{2}\right), f_{0}^{-1}\left(V_{1}\right)\right)$. When $\left(V_{2}, V_{1}\right)$ is a pair of free complete intersections, we show that $\mathcal{K}_{V_{2}, V_{1}}$ has a Cohen-Macaulay reduction, deducing that the discriminant for this equivalence is a free* divisor (Theorem 5), although it need not be free.

An important special case occurs when $V_{2}=\mathbb{C}^{p_{1}}$ so $X$ is an ICIS and $V_{1}, 0 \subset$ $\mathbb{C}^{p_{1}}, 0$ is a free divisor. Provided $V_{1}$ generically has Morse type singularities, the 
$\mathcal{K}_{\mathbb{C}^{p_{1}, V_{1}}}$-discriminant is a free divisor (Theorem 6). In particular, this yields the freeness of discriminants for versal unfoldings of pairs which consist of the zero set of a function germ on an ICIS, or an ICIS together with its intersection with an almost free divisor such as: nonlinear generic arrangements of isolated hypersurface singularities, discriminants of finitely determined map germs, etc.

Third, in the second part of $\S 3$, we consider the collection of equivalences $\mathcal{K}(V)$, $\mathcal{A}(V)$ or $\mathcal{R}^{+}(V)$ of mappings on a fixed free complete intersection $V$. This is iii) in Table 1 . Now we only obtain general results about when discriminants are free* divisors. We give a CM-reduction for $\mathcal{K}(V)$ and deduce Theorem 7 that $\mathcal{K}(V)-$ discriminants for versal unfoldings of ICIS germs on $V$ are free* divisors. From this we deduce free* divisor structures for the discriminants for $\mathcal{A}(V)$-stable germs (Theorem 8) and $\mathcal{R}^{+}(V)$-versal unfoldings (Corollary 9).

We actually prove these results in a different order, considering the hardest cases iv) and v) of Table 1 first in $\S 4$ and $\S 5$. This allows us to outline arguments in later sections by referring to the methods of these proofs. The case i) of Table 1 will be covered in $\S 6$ and $\S 7$, and iii) of Table 1 , in $\S 8$.

In $\S 9$, we conclude by briefly discussing the implications of the results on free divisors for understanding the discriminants of versal unfoldings. The author would like to thank the referee for a number of very valuable comments, including raising some of the questions in $\S 9$.

\section{Preliminaries}

Notation and Terminology from Parts I and II. We begin by recalling several key ideas from parts I and II [D5] and [D6] and [D2] which are central to the results obtained here. See these references for further details.

$\mathcal{G}$-liftable vector fields and $\mathcal{G}$-discriminants. All of the groups of equivalences $\mathcal{G}$ we consider are geometric subgroups of $\mathcal{A}$ or $\mathcal{K}$ for the category of holomorphic germs. For such groups we have the basic theorems of singularity theory such as the finite determinacy and versality theorems (see [D3]). For such a group there is an action of $\mathcal{G}$ on a space of germs $\mathcal{F}$, where in the examples we consider $\mathcal{F}=\mathcal{C}(n, p)$ is the space of holomorphic germs $f: \mathbb{C}^{n}, 0 \rightarrow \mathbb{C}^{p}, 0$. There is also a corresponding action of the group of unfoldings $\mathcal{G}_{\text {un }}(q)$ on the space of unfoldings $\mathcal{F}_{\text {un }}(q)$ on $q$ parameters, for all integer $q \geq 0$. These actions satisfy four conditions given in [D3]. For an unfolding $F \in \mathcal{F}_{\text {un }}(q)$, we have the orbit map $\alpha_{F}: \mathcal{G}_{\text {un }}(q) \rightarrow \mathcal{F}_{\text {un }}(q)$ and the corresponding infinitesimal orbit map $d \alpha_{F}: T \mathcal{G}_{\text {un,e }}(q) \rightarrow T \mathcal{F}_{\text {un,e }}(q)$ (for $q=0$ and a germ $f_{0}$ this is denoted $\left.d \alpha_{f_{0}}: T \mathcal{G}_{e} \rightarrow T \mathcal{F}_{e}\right)$. A germ of a vector field $\zeta \in \theta_{q}$ is said to be $\mathcal{G}$-liftable if there is a $\xi \in T \mathcal{G}_{\text {un,e }}(q)$ such that $d \alpha_{F}(\xi+\zeta)=0$. Let $\mathcal{L}_{\mathcal{G}}(F)$ denote the $\mathcal{O}_{\mathbb{C}^{q}, 0}$-module of $\mathcal{G}$-liftable vector fields.

The orbit tangent spaces $T \mathcal{G}_{\text {un,e }} \cdot F$ (resp. $T \mathcal{G}_{e} \cdot f_{0}$ ) are the images of $d \alpha_{F}$ (resp. $d \alpha_{f_{0}}$ ) and the normal spaces $N \mathcal{G}_{\text {un }, e} \cdot F$ (resp. $N \mathcal{G}_{e} \cdot f_{0}$ ) are the cokernels. For a germ $f_{0}$ of finite $\mathcal{G}$-codimension with unfolding $F$ on $q$ parameters, $N \mathcal{G}_{\text {un }, e} \cdot F$ is a finitely generated $\mathcal{O}_{\mathbb{C}^{q}, 0}$-module. The $\mathcal{G}$-discriminant is the complex analytic set $D_{\mathcal{G}}(F)=\operatorname{supp}\left(N \mathcal{G}_{\text {un }, e} \cdot F\right)$ (as an $O_{\mathbb{C}^{q}, 0}$-module). This set could equally well be thought of as a bifurcation set. However, results from part I show that often bifurcation sets can be reinterpreted as discriminants so we retain the term " $\mathcal{G}$ discriminant" understood in this more general sense. A group $\mathcal{G}$ is said to have geometrically defined discriminants if for a $\mathcal{G}$-versal unfolding $F$, the flow generated 
by a $\mathcal{G}$-liftable vector field $\zeta$ preserves $D_{\mathcal{G}}(F)$. All standard examples have this property.

$\operatorname{Derlog}(V)$ and $\mathcal{K}_{V}$-equivalence. A basic group on which many others are based is $\mathcal{K}_{V^{-}}$equivalence of nonlinear sections $f_{0}: \mathbb{C}^{n}, 0 \rightarrow \mathbb{C}^{p}, 0$ of a germ of an analytic set $V, 0 \subset \mathbb{C}^{p}, 0$, which captures the ambient equivalence of $V_{0}=f_{0}^{-1}(V)$. The properties of the nonlinear sections are determined by $\operatorname{Derlog}(V)$, the module of vector fields $\zeta$ on $\mathbb{C}^{p}, 0$ which are tangent to $V$, i.e. $\zeta(I(V)) \subset I(V)$ for the ideal $I(V)$ of germs vanishing on $V$. The classes of $V$ whose sections give the analogues of ICIS are the free divisors ( $\operatorname{Derlog}(V)$ is a free module of rank $p$ ) and free complete intersections $(\operatorname{Der} \log (h)$ is a free module for the defining equation $h$ of $V$, where $\operatorname{Derlog}(h)$ is the module of vector fields $\zeta$ on $\mathbb{C}^{p}, 0$ such that $\left.\zeta(h)=0\right)$. Evaluating these modules at a point $y \in \mathbb{C}^{p}$ gives the logarithmic tangent spaces $T_{\text {log }}(V)_{(y)}$ and $T_{l o g}(h)_{(y)}$. These can be used in place of $T_{y} S$, the tangent space of the stratum of the canonical Whitney statification of $V$. The codimensions of the sets off which $T_{y} S=T_{l o g}(V)_{(y)}\left(\operatorname{resp} . T_{l o g}(h)_{(y)}\right)$ are denoted by $h n(V)$ (resp. $\left.h(V)\right)$. We can consider transversality to $V$ at $y$ either using $T_{y} S$ (geometric transversality) or $T_{l o g}(V)_{(y)}$ (algebraic transversality).

We weaken the notion of freeness for divisors by allowing $V$ to be stabilized to $V^{\prime}=V \times \mathbb{C}^{r}$ and replacing $\operatorname{Derlog}\left(V^{\prime}\right)$ by a free submodule $M$ of rank $p+r$ which defines $V^{\prime}$ with a nonreduced structure. We denote the module $M$ by $\operatorname{Derlog}^{*}(V)$ and refer to $V$ as being a free* divisor defined by $M$. This is highly non-unique, so we seek an $M$ which captures, as well, geometric properties of $V$.

Cohen-Macaulay properties of groups $\mathcal{G}$. For us, a key algebraic property of $\mathcal{G}$ is its being Cohen-Macaulay, which means: $\mathcal{G}$ has geometric defined discriminants, and for each versal unfolding $F \in \mathcal{F}_{\text {un }}(q), N \mathcal{G}_{\text {un,e }} \cdot F$ is Cohen-Macaulay as a $\mathcal{O}_{\mathbb{C}^{q}, 0}$-module, with $\operatorname{supp}\left(N \mathcal{G}_{\text {un }, e} \cdot F\right)$ of dimension $q-1$.

For a group $\mathcal{G}$ with geometrically defined discriminants, a Cohen-Macaulay reduction (CM-reduction) is a geometric subgroup $\mathcal{G}^{*}$ which is Cohen-Macaulay and satisfies: $f \in \mathcal{F}$ has finite $\mathcal{G}^{*}$-codimension iff it has finite $\mathcal{G}$-codimension; and for an unfolding $F$ of a finite $\mathcal{G}$-codimension germ,

$$
\operatorname{supp}\left(N \mathcal{G}_{u n, e}^{*} \cdot F\right)=\operatorname{supp}\left(N \mathcal{G}_{\text {un,e }} \cdot F\right)
$$

This support is the $\mathcal{G}$-discriminant of $F$, denoted $D_{\mathcal{G}}(F)$. In all of the examples we consider, if $\mathcal{G}^{*}$ is the $\mathrm{CM}$-reduction of $\mathcal{G}$, then we shall let $\operatorname{Derlog}^{*}\left(D_{\mathcal{G}}(F)\right) \stackrel{\text { def }}{=}$ $\mathcal{L}_{\mathcal{G}^{*}}(F)$, the module of $\mathcal{G}^{*}$-liftable vector fields. For example, if $V$ is a free divisor, $\mathcal{K}_{V}$ is Cohen-Macaulay (by results of part I); while if $V$ is a free complete intersection $\mathcal{K}_{V}$ is not in general Cohen-Macaulay but it does have the CM-reduction [D6, Theorem 4] $\mathcal{K}_{V}^{*}$, which consists of diffeomorphisms in $\mathcal{K}_{V}$ whose restrictions to $V \times \mathbb{C}^{n}$ equal restrictions of elements of $\mathcal{K}_{h}$ (diffeomorphisms which preserve the level sets of the defining equation $h$ for $V$ (see [D6, $\S 6]$ ).

To apply the main theorems of part II, we provide a criterion for genericity of $\mathcal{G}$-liftable vector fields resulting from genericity of Morse-type singularities.

Morse-type singularities for $\mathcal{K}_{V}$-equivalence. Given an analytic germ $V, 0 \subset \mathbb{C}^{p}$, a map germ $g: \mathbb{C}^{n}, 0 \rightarrow \mathbb{C}^{p}, 0$ is a Morse-type singularity in dimension $n$ for $\mathcal{K}_{V^{-}}$ equivalence if $g$ has $\mathcal{K}_{V, e^{-}}$-odim $=1$ and is $\mathcal{K}_{V^{-}}$-equivalent to a germ $f_{0}$ such that for a common choice of local coordinates both $f_{0}$ and $V$ are weighted homogeneous. By part I [D5] or Lemma 7.3 of part II [D6], a Morse-type singularity can be 
put into normal form via $\mathcal{K}_{V}$-equivalence. We may assume $V, 0=\mathbb{C}^{r} \times V_{0}, 0$ for $V_{0}, 0 \subset \mathbb{C}^{p^{\prime}}, 0$, and with respect to coordinates for which $V_{0}, 0$ is weighted homogeneous, $f_{0}$ has the local normal form

$$
f_{0}\left(x_{1}, \ldots, x_{n}\right)=\left(0, \ldots, 0, x_{1}, \ldots, x_{p^{\prime}-1}, \sum_{j=p^{\prime}}^{n} x_{j}^{2}\right)
$$

with a $\mathcal{K}_{V}$-versal unfolding

$$
F(x, u)=\left(f_{0}(x)+(0, \ldots, 0, u), u\right)
$$

The sign of weight $\operatorname{wt}\left(y_{p}\right)>,<$, or $=0$ is called the exceptional weight type; and (by Lemma 4.10 of part I or Lemma 7.8 of part II) $u \frac{\partial}{\partial u}$ is $\mathcal{K}_{V}$-liftable.

The notion of Morse-type singularity extends to $y \in V$ using $\mathcal{K}_{(V, y)}$-equivalence. Then, $V$ is said to generically have Morse-type singularities in dimension $n$ if: all points on the canonical Whitney stata of $V$ of codimension $\leq n+1$ have Morse-type singularities of nonzero exceptional weight type; and any stratum of codimension $>n+1$ lies in the closure of a stratum of codimension $=n+1$.

Genericity of $\mathcal{G}^{*}$-liftable vector fields. For a general equivalence $\mathcal{G}$, it is more difficult to describe the analogues of Morse-type singularities and deduce their genericity. This can effectively be done for $\mathcal{G}^{*}$, a CM-reduction of $\mathcal{G}$ through the notion of $\mathcal{G}$ generically having $\mathcal{G}^{*}$-liftable vector fields. This means that for a $\mathcal{G}$ versal unfolding $F$ on $q$ parameters, there is a Zariski open subset $\mathcal{Z}$ of $D_{\mathcal{G}}(F)_{\text {reg }}$ having nonempty intersection with all irreducible components so that at points $u \in \mathcal{Z}:(1) h \cdot \Theta_{q} \subset \mathcal{L}_{\mathcal{G}^{*}}(F)$ as sheaves on $\mathcal{Z}$ and $(2) T_{\text {log }^{*}}\left(D_{\mathcal{G}}(F)_{(u)}=T_{u} D_{\mathcal{G}}(F)_{\text {reg }}\right.$.

For $V, 0 \subset \mathbb{C}^{p}, 0$ the group $\mathcal{K}_{V}$ acts on $\mathcal{C}(n, p)$ for $n$ and $p$ fixed. For $y \in V$ in a neighborhood of 0 , we have a local form $\mathcal{K}_{(V, y)}$ for $\mathcal{K}_{V}$-equivalence of germs with target $y$. We consider $\mathcal{G}$ a geometric subgroup of $\mathcal{K}_{V}$ which we suppose has analogous local forms. Then, we can apply the following criterion to verify that $\mathcal{G}$ generically has $\mathcal{G}^{*}$-liftable vector fields.

\section{(1.3) Criterion for genericity of $\mathcal{G}^{*}$-liftable vector fields:}

(1) There is a local form of $\mathcal{G}^{*}$-equivalence at each $y$, and the module of $\mathcal{G}^{*}$ liftable vector fields restricts to the module of locally liftable vector fields for the local $\mathcal{G}^{*}$-equivalence for all $y$ in a neighborhood of 0 .

(2) $V$ generically has Morse-type singularities in dimension $n$.

(3) There is a Zariski open subset $\mathcal{U}$ of jets algebraically transverse to $V$ and have $\mathcal{G}_{e}^{*}$-codim 0 for the local form of $\mathcal{G}^{*}$-equivalence at the target point $y$.

(4) There is a Zariski open subset $\mathcal{W}$ of the set of jets not algebraically transverse to $V$ such that for each point $y$ in a stratum of $V$ of codimension $\leq n+1, \mathcal{W}$ is dense in the fiber over $y$ and : i) any $z \in \mathcal{W}$ is the jet of a Morse-type singularity; ii) it has $\mathcal{G}_{e}^{*}$-codim 1 and is $\mathcal{G}^{*}$-equivalent to the local normal form (1.1) and $u \frac{\partial}{\partial u}$ is $\mathcal{G}^{*}$-liftable for the versal unfolding $F$ in (1.2).

Proposition 1.1. If $\mathcal{G}$ is a geometric subgroup of $\mathcal{K}_{V}$ with $C M$-reduction $\mathcal{G}^{*}$ which satisfies the criterion (1.3), then $\mathcal{G}$ generically has $\mathcal{G}^{*}$-liftable vector fields. 
Proof. Let $F$ be a $\mathcal{G}^{*}$-versal unfolding of $f_{0}$. As $\mathcal{G} \subseteq \mathcal{K}_{V}, F$ is also a $\mathcal{K}_{V}$-versal unfolding. Any irreducible component of $D_{\mathcal{G}}(F)=D_{\mathcal{G}^{*}}(F)$ contains a Zariski open subset of parameter values $u$ such that $\bar{F}(x, u)$ misses strata of $V$ of codimension $>n+1$ (as this is true for a $\mathcal{K}_{V}$-versal unfolding by (2) of (1.3) and the results of Part I). Again arguing as in Part I and using (3), we may perturb $f_{0}$ so it only has a single Morse-type singularity. A further perturbation will ensure that its jet belongs to $\mathcal{W}$ for that $y$. Hence, by (4), the single Morse-type singularity is $\mathcal{G}^{*}-$ equivalent to the local normal form (1.1) and $u \frac{\partial}{\partial u}$ is $\mathcal{G}^{*}$-liftable for the unfolding $F$ in (1.2).

Thus, the regular parts of the irreducible components contain nonempty Zariski open subsets consisting of a single Morse-type singularity which has $\mathcal{G}_{e}^{*}$-codim 1 for the local $\mathcal{G}^{*}$-equivalence and locally for the normal coordinate $u, u \frac{\partial}{\partial u}$ is $\mathcal{G}^{*-}$ liftable. Thus, the discriminant for the local $\mathcal{G}^{*}$-equivalence near such a $y$ is a smooth hypersurface contained in $D_{\mathcal{G}^{*}}(F)$ and hence agrees with it near $y$. This implies (2) of the definition for genericity of $\mathcal{G}^{*}$-liftable vector fields as all of the tangent vector fields are locally $\mathcal{G}^{*}$-liftable near $y$. Together with $u \frac{\partial}{\partial u}$ being $\mathcal{G}^{*-}$ liftable, this implies that locally all of $h \cdot \Theta_{p(y)}$ is $\mathcal{G}^{*}$-liftable (with $h$ the defining equation for $D_{\mathcal{G}}(F)$ ). Finally, using (1) of (1.3), we conclude property (1) for the genericity of $\mathcal{G}^{*}$-liftable vector fields.

\section{Discriminants for Equivalences Preserving Free Divisors in Source}

Let $V, 0 \subset \mathbb{C}^{n}, 0$ be a germ of a free divisor. We consider the equivalences of map germs $f_{0}: \mathbb{C}^{n}, 0 \rightarrow \mathbb{C}^{m}, 0$ preserving $V$ in the source: ${ }_{V} \mathcal{A},{ }_{V} \mathcal{K}$, or ${ }_{V} \mathcal{R}^{+}$(if $m=1$ ), as given in Table 1 (as already mentioned, these are geometric subgroups of $\mathcal{A}$ or $\mathcal{K})$.

First, consider ${ }_{V} \mathcal{K}$ equivalence. The ${ }_{V} \mathcal{K}$-discriminant for a versal unfolding $F(x, u)=\left(F_{u}(x), u\right)$ of $f_{0}$ is given by

$$
\begin{aligned}
& D_{V} \mathcal{K}(F)=\left\{u \in \mathbb{C}^{q}: X_{u}=f_{u}^{-1}(0)\right. \text { is not smooth or } \\
& \left.\qquad X_{u} \text { is not algebraically transverse to } V\right\}
\end{aligned}
$$

The first theorem expresses conditions that $D_{V} \mathcal{K}(F)$ is a free divisor in terms of the dimension $n-m$ and $V$ generically having Morse-type singularities in dimension $n-m$.

Theorem 1. Suppose that $V$ is a free divisor and that $f_{0}: \mathbb{C}^{n}, 0 \rightarrow \mathbb{C}^{m}, 0, n \geq m$, has finite ${ }_{V} \mathcal{K}$-codimension with versal unfolding $F$. If $n-m<h n(V)$, then ${ }_{V} \mathcal{K}$ is Cohen-Macaulay so the discriminant $D_{V} \mathcal{K}(F)$ is a free* divisor defined by

$$
\operatorname{Derlog}^{*}\left(D_{V} \mathcal{K}(F)\right)=\text { module of }{ }_{V} \mathcal{K} \text {-liftable vector fields. }
$$

If moreover $V$ generically has Morse-type singularities in dimension $n-m$ then $D_{V} \mathcal{K}(F)$ is a free divisor.

Next, we can apply the preceding to discriminants of ${ }_{V} \mathcal{A}$-stable germs using the relation between ${ }_{V} \mathcal{K}$-versal unfoldings and ${ }_{V} \mathcal{A}$-stable germs analogous to that for $\mathcal{K}$ and $\mathcal{A}$ due to Mather [M-IV] or Martinet [Mar]. If $f_{0}: \mathbb{C}^{n}, 0 \rightarrow \mathbb{C}^{m}, 0$ is a stable germ for ${ }_{V} \mathcal{A}$-equivalence, then $F(x, y)=(\bar{F}(x, y), y)$, with $\bar{F}(x, y)=f(x)-y$ is 
a ${ }_{V} \mathcal{K}$-versal unfolding of $f_{0}$ and the projection $\pi: \bar{F}^{-1}(0), 0 \rightarrow \mathbb{C}^{m+q}, 0$ can be identified with $f$ (see Lemma 7.3).

The ${ }_{V} \mathcal{A}$-discriminant of a germ $f_{0}: \mathbb{C}^{n} \rightarrow \mathbb{C}^{m}$ is defined to be

$$
D_{V} \mathcal{A}\left(f_{0}\right)=\left\{y \in \mathbb{C}^{m}: \text { there is } x \in f^{-1}(y) \text { with } d f_{0}(x)\left(T_{\text {log }}(V)_{(x)} \neq T_{y} \mathbb{C}^{m}\right\}\right.
$$

A condition for freeness of $D_{V} \mathcal{A}\left(f_{0}\right)$ for stable $f_{0}$ takes the following form.

Theorem 2. Suppose that $V, 0 \subset \mathbb{C}^{n}, 0$ is a free divisor and that $f_{0}: \mathbb{C}^{n}, 0 \rightarrow \mathbb{C}^{m}, 0$, $n \geq m$, is ${ }_{V} \mathcal{A}$-stable. Provided $n-m<h n(V)$, the ${ }_{V} \mathcal{A}$-discriminant $D_{V} \mathcal{A}(F)$ is a free* divisor defined by

$$
\operatorname{Derlog}^{*}\left(D_{V} \mathcal{A}\left(f_{0}\right)\right)=\text { module of }{ }_{V} \mathcal{A} \text {-liftable vector fields for } f_{0}
$$

If moreover $V$ generically has Morse-type singularities in dimension $n-m$, then the ${ }_{V} \mathcal{A}$-discriminant is a free divisor.

Third, we obtain results for ${ }_{V} \mathcal{R}^{+}$-equivalence. A versal unfolding $F(x, u)=$ $\left(F_{u}(x), u\right): \mathbb{C}^{n+q}, 0 \rightarrow \mathbb{C}^{p+q}, 0$ of a germ $f_{0}$ under ${ }_{V} \mathcal{R}^{+}$-equivalence is also versal for ${ }_{V} \mathcal{A}$-equivalence. For ${ }_{V} \mathcal{R}^{+}$, the discriminant of $F$ viewed as a map germ is

$\left\{(y, u) \in \mathbb{C}^{1+q}: F_{u}^{-1}(y)\right.$ is not smooth or is not algebraically transverse to $\left.V\right\}$

Then, for ${ }_{V} \mathcal{R}^{+}$-equivalence, there is the following.

Corollary 3. Suppose that $V$ is a free divisor which generically has Morse-type singularities in dimension $n-1$. Let $f_{0}: \mathbb{C}^{n}, 0 \rightarrow \mathbb{C}, 0$ have finite ${ }_{V} \mathcal{R}^{+}$-codimension with versal unfolding $F$. If $n-1<h n(V)$, then the ${ }_{V} \mathcal{R}^{+}$-discriminant of the map germ $F$ is a free divisor defined by the module of ${ }_{V} \mathcal{A}$-liftable vector fields.

For $f_{0}$ of finite ${ }_{V} \mathcal{A}$-codimension, we may also consider the ${ }_{V} \mathcal{A}$-bifurcation set of an $V_{\mathcal{A}} \mathcal{A}$-versal unfolding $F$ of $f_{0}$. We ask when the results on the freeness of bifurcation sets for hypersurface germs in $[\mathrm{Br}]$ and $[\mathrm{To}]$ and more general map germs in [D5] extend to ${ }_{V} \mathcal{A}$-equivalence. The bifurcation set is defined by

$$
B_{V} \mathcal{A}(f)=\left\{u \in \mathbb{C}^{q}: F_{u} \text { is not }{ }_{V} \mathcal{A} \text {-stable }\right\} .
$$

We need the analogue of a distinguished bifurcation class in [D5, Def.6.1].

Definition 2.1. For a free divisor $V, 0 \subset \mathbb{C}^{n}, 0$, map germs $f_{0}: \mathbb{C}^{n}, 0 \rightarrow \mathbb{C}^{p}, 0$ belong to a $V \mathcal{A}$-distinguished bifurcation class if : they belong to the distinguished bifurcation class in the sense of (6.1) of [D5]; and, in addition, for all $x \in V$ in a neighborhood of 0 , with ${ }_{V} \mathcal{K}$-equivalence for germs at $x$ denoted by ${ }_{(V, x)} \mathcal{K}$

(1) all $(V, x) \mathcal{K}$-classes of extended codimension $>p+1$ lie in the closure of $(V, x) \mathcal{K}$-classes of extended codimension $p+1$;

(2) all ${ }_{(V, x)} \mathcal{K}$-classes of extended codimension $<p+1$ contain germs of ${ }_{(V, x)} \mathcal{A}_{e^{-}}$ codimension 1.

(3) all ${ }_{(V, x)} \mathcal{K}$-classes of extended codimension $<p+1$ are ${ }_{(V, x)} \mathcal{K}$-simple germs.

Theorem 4. Suppose that $V$ is a free divisor which generically has Morse-type singularities in dimension $n-p$, with $n-p<h n(V)$. Suppose $f_{0}: \mathbb{C}^{n}, 0 \rightarrow \mathbb{C}^{p}, 0$ belongs to the ${ }_{V} \mathcal{A}$-distinguished bifurcation class and has finite ${ }_{V} \mathcal{A}$-codimension. Then, for the versal unfolding $F$ of $f_{0}$, the bifurcation set $B_{V \mathcal{A}}(F)$ is a free divisor with

$$
\operatorname{Derlog}\left(B_{V \mathcal{A}}(F)\right)=\text { module of }{ }_{V} \mathcal{A} \text {-liftable vector fields. }
$$

The proofs of these theorems will be given in $\S 6$ and $\S 7$. 
Example 2.2. Let $V, 0 \subset \mathbb{C}^{2}, 0$ be the free divisor defined by $y x^{2}-y^{3}=0$. We consider the projection map $f_{0}(x, y)=x$ under ${ }_{V} \mathcal{K}$-equivalence. The ${ }_{V} \mathcal{K}$-versal unfolding is given by $f\left(x, u_{1}, u_{2}\right)=\left(x+u_{1} y+u_{2}, u_{1}, u_{2}\right)$ and the ${ }_{V} \mathcal{K}$-discriminant is the $u_{2}$-axis. However, $u_{2}$ is a modulus which reflects the cross ratio of the three lines $f_{0}^{-1}(0)$ together with the $y$-axis; hence the vector field $\frac{\partial}{\partial u_{2}}$ is not ${ }_{V} \mathcal{K}$-liftable (although $u_{1} \frac{\partial}{\partial u_{2}}$ is). Thus, the module of ${ }_{V} \mathcal{K}$-liftable vector fields does not define $D_{V} \mathcal{K}(f)$ as a free divisor, but rather only a free* divisor. This is the counterpart of the example for nonlinear sections of free divisors given in Part I (at the end of $\S 9$ in [D5]).

We consider the consequences of Theorems 1-4 to the various examples mentioned earlier.

Discriminants for Equivalence of ICIS Preserving a Singular Divisor in the Source. We consider consequences for equivalences of germs of holomorphic functions preserving a free divisor in the source $V, 0 \subset \mathbb{C}^{n}, 0$.

\section{(2.3) Germs on Manifolds with Boundaries and Corners}

Let $V=A_{k} \times \mathbb{C}^{n-k}$, where $A_{k} \subset \mathbb{C}^{k}$ denotes the Boolean arrangement consisting of the coordinate hyperplanes. The corresponding real version of $V$ is a general boundary or corner. The important special property of Boolean arrangements, and hence such $V$, is that they generically have Morse-type singularities in all dimensions and are holonomic so $h n(V)\left(=h n\left(A_{k}\right)\right)=\infty$ (see [D5, Lemma 7.7]. Hence, the preceding results apply to them without restriction.

We draw the following conclusions for germs $f_{0}: \mathbb{C}^{n}, 0 \rightarrow \mathbb{C}^{p}, 0$.

(1) If $f_{0}$ has finite ${ }_{V} \mathcal{K}$-codimension with ${ }_{V} \mathcal{K}$-versal unfolding $F$, then by Theorem 1 the ${ }_{V} \mathcal{K}$-discriminant of $F$ is a free divisor.

(2) Moreover, if $f_{0}$ is ${ }_{V} \mathcal{A}$-stable then by Theorem 2, the ${ }_{V} \mathcal{A}$-discriminant of $f_{0}$ is a free divisor.

(3) Hence, for holomorphic germs $f_{0}: \mathbb{C}^{n}, 0 \rightarrow \mathbb{C}, 0$ on manifolds with boundaries and corners, considered by Siersma [Si], Arnold [A2], Lyashko [Ly], and Goryunov [Go2] (for $\mathbb{C}^{2}$ ), the ${ }_{V} \mathcal{K}$ or ${ }_{V} \mathcal{R}^{+}$-discriminants of the appropriate versal unfoldings are free divisors.

(4) Also, finite ${ }_{V} \mathcal{A}$-codimension germs $f_{0}: \mathbb{C}^{n}, 0 \rightarrow \mathbb{C}, 0$ belong to the ${ }_{V} \mathcal{A}$ distinguished bifurcation class of Def. 2.1 (both conditions ii) and iii) are immediate and i) can be easily checked using methods of [D5, §4]). Thus, by Theorem 4 , the ${ }_{V} \mathcal{A}$-bifurcation sets are free divisors.

(5) Lastly, for holomorphic germs of "projection mappings "of manifolds with boundaries and corners $f_{0}: \mathbb{C}^{n}, 0 \rightarrow \mathbb{C}^{2}, 0$ (considered in the real case by by Bruce-Giblin [BG1] for $n=2$ and Tari [Ta] for $n=3$ ), both the ${ }_{V} \mathcal{K}-$ discriminants of versal unfoldings and the ${ }_{V} \mathcal{A}$-discriminants of ${ }_{V} \mathcal{A}$-stable projections are free divisors.

\section{(2.4) Germs on Manifolds with Singular Boundaries}

Next, suppose we allow more general "singular boundaries", which are free divisors $V, 0 \subset \mathbb{C}^{n}, 0$. There are still a number of important cases where $V$ generically has Morse-type singularities. Two such cases are: 1) $V, 0$ is a discriminant of a stable germ $V=D(F)$, where $F$ is the versal unfolding of a germ in the distinguished 
bifurcation class defined in [D5, Def. 6.1]; and 2) $V=\mathcal{C}_{k} \times \mathbb{C}^{n-2}$ is a higher order cusp hypersurface for $\mathcal{C}_{k} \subset \mathbb{C}^{2}$ a higher order cusp defined by $x_{1}^{2}-x_{2}^{k}=0$.

We consider a finite ${ }_{V} \mathcal{K}$-codimension germ $f_{0}: \mathbb{C}^{n}, 0 \rightarrow \mathbb{C}^{p}, 0$ for $n \geq p$, with ${ }_{V} \mathcal{K}$-versal unfolding $F$. If $V=D(F)$ we suppose $n-p<h n(D(F))$, while for $V$ a higher order cusp hypersurface $h n(V)=\infty$ so there is no other condition on $n-p$. Then, again by Theorem 1 the ${ }_{V} \mathcal{K}$-discriminant of $F$ is a free divisor. Also, if $f_{0}$ is ${ }_{V} \mathcal{A}$-stable then by Theorem 2, the ${ }_{V} \mathcal{A}$-discriminant of $f_{0}$ is a free divisor. In the special case of function germs $f_{0}: \mathbb{C}^{n}, 0 \rightarrow \mathbb{C}, 0$, we deduce that the ${ }_{V} \mathcal{R}^{+}$-discriminant of $F$ is a free divisor.

These results apply to both function germs $f_{0}: \mathbb{C}^{n}, 0 \rightarrow \mathbb{C}, 0$ for singular boundaries $V$ which are the discriminants of stable $A_{k}$ singularities considered by Arnold [A1] (now $A_{k}$ refers to the Morin singularities not the Boolean arrangements), and to function germs $f_{0}: \mathbb{C}^{2}, 0 \rightarrow \mathbb{C}, 0$ with $V$ a (higher order) cusp singularity considered by Lyashko [Ly]. The ${ }_{V} \mathcal{K}$-discriminants or ${ }_{V} \mathcal{A}$-discriminants of versal unfoldings of such germs are free divisors.

However, the freeness fails as soon as we consider functions when the singular boundary $V$ no longer generically has Morse-type singularities. This already occurs for $V=\mathcal{C} \times \mathbb{C}^{k}$ for $\mathcal{C}, 0 \subset \mathbb{C}^{2}$ an isolated curve singularity other than an $A_{1}$ or higher cusp $A_{k}$ singularity.

\section{(2.5) Germs on Manifolds with General Free Boundaries}

Third, we consider the equivalence of germs $f_{0}: \mathbb{C}^{n}, 0 \rightarrow \mathbb{C}^{p}, 0, n \geq p$, preserving a singular boundary $V, 0 \subset \mathbb{C}^{n}$, where $V$ is a free divisor, but without assuming genericity of Morse-type singularities in dimension $n-p$. Examples include: an arbitrary free arrangement of hyperplanes, a discriminant of a stable germ outside the distinguished bifurcation class in $\S 6$ of Part I, bifurcation sets, etc. Provided $n-p<h n(V)$, then applying Theorems $1-3$, we can still conclude that a ${ }_{V} \mathcal{K}$-versal unfolding of $f_{0}$ still has ${ }_{V} \mathcal{K}$-discriminant which is a free* divisor (with a similar conclusion for ${ }_{V} \mathcal{R}^{+}$-equivalence if $p=1$ ). Also, the ${ }_{V} \mathcal{A}$-discriminant will be a free* divisor if $f_{0}$ is $V \mathcal{A}$-stable. We demonstrated in example 2.2 that the more limited conclusion is unavoidable.

\section{Discriminants of Mappings and Divisors on Complete Intersections}

The second situation we consider concerns a divisor or mapping on a complete intersection, where we allow the complete intersection to be fixed or vary. Unlike the previous section, the equivalence will only capture the properties on the complete intersection, ignoring behavior off of it.

We first consider the case of a divisor on a complete intersection, where both vary. An important special case concerns a divisor which is the intersection of an almost free divisor with an ICIS. Third, we describe analogous results for a mapping on a fixed free complete intersection $V$.

Relative Complete Intersections. For the relative case of a divisor on a complete intersection we allow both to vary. We consider a pair $V_{1}, 0 \subset V_{2}, 0 \subset \mathbb{C}^{p}, 0$, of germs of subvarieties, which we denote by $\left(V_{2}, V_{1}\right)$. We define the equivalence of such pairs via $\mathcal{K}_{V_{2}, V_{1}}$-equivalence, which is the analogue of $\mathcal{K}_{V}$-equivalence. It is the subgroup of $\mathcal{K}$ consisting of germs of diffeomorphisms of $\mathbb{C}^{n} \times \mathbb{C}^{p}, 0$ which perserve each of the $\mathbb{C}^{n} \times V_{i}, i=1,2$ (see [D1] for precise definition). Just as $\mathcal{K}_{V}$-equivalence captures the equivalence of the germs of varieties $f_{0}^{-1}(V)$, so too 
the equivalence $\mathcal{K}_{V_{2}, V_{1}}$ captures the equivalence of the pair of germs of varieties $\left(f_{0}^{-1}\left(V_{2}\right), f_{0}^{-1}\left(V_{1}\right)\right)$. By the same arguments for $\mathcal{K}_{V}$ [D1], $\mathcal{K}_{V_{2}, V_{1}}$ is a geometric subgroup of $\mathcal{A}$ or $\mathcal{K}$, so the basic theorems of singularity theory apply to it.

Remark 3.1. If the $V_{i}$ form a pair of increasing linear subspaces $\mathbb{C}^{p_{1}} \subset \mathbb{C}^{p_{2}}$ then $\mathcal{K}_{V_{2}, V_{1}}$-equivalence is the equivalence of contact to a pair of flags as in $[\mathrm{K}]$, [Zl], and $[\mathrm{Zl} 2]$. This is also a type of ladder of mappings as in [D4, §8]

Definition 3.2. The pair $V_{1}, 0 \subset V_{2}, 0 \subset \mathbb{C}^{p}, 0$, will be called a free complete intersection pair if $V_{2}=\mathbb{C}^{p_{1}} \times W_{2}$ and $V_{1}=W_{1} \times W_{2}$, where $W_{1}, 0 \subset \mathbb{C}^{p_{1}}, 0$ is a free divisor and $W_{2}, 0 \subset \mathbb{C}^{p_{2}}, 0$ is a free complete intersection.

We then consider pairs obtained as pullbacks by a section $f_{0}: \mathbb{C}^{n}, 0 \rightarrow \mathbb{C}^{p}, 0$ of a free complete intersection pair $\left(V_{2}, V_{1}\right)$, where we require $f_{0}$ to be algebraically transverse off 0 to both $V_{1}$ and $V_{2}$. If we represent $f_{0}=\left(f_{01}, f_{02}\right)$ under the decomposition $\mathbb{C}^{p}=\mathbb{C}^{p_{1}} \times \mathbb{C}^{p_{2}}$, then $f_{0}$ algebraically transverse to $V_{2}$ implies $f_{02}$ is algebraically transverse to $W_{2}$; and similarly $f_{0}$ algebraically transverse to $V_{1}$ implies $f_{01}$ is algebraically transverse to $W_{1}$. Then, $X=f_{0}^{-1}\left(V_{2}\right)=f_{02}^{-1}\left(W_{2}\right)$ is an "almost free complete intersection", and likewise, $f_{01}^{-1}\left(W_{1}\right)$ is an almost free divisor. In addition, $f_{0}$ being algebraically transverse to $V_{1}$ off 0 implies that $X_{0}=f_{0}^{-1}\left(V_{1}\right)$ is the transverse intersection off 0 of $f_{01}^{-1}\left(W_{1}\right)$ with $X$ (see [D2, $\left.\S 7\right]$ ). The intersection is again an almost free complete intersection.

For a versal unfolding $F(x, u)=\left(F_{u}(x), u\right)$ of $f_{0}$, the $\mathcal{K}_{V_{2}, V_{1}}$-discriminant can be described geometrically as follows.

$D_{V_{2}, V_{1}}(F)=\left\{u \in \mathbb{C}^{q}: F_{u}\right.$ fails to be (algebraically) transverse to either $V_{1}$ or $\left.V_{2}\right\}$

A special case will be the free complete intersection pair $V_{1} \subset \mathbb{C}^{p_{1}} \subset \mathbb{C}^{p}$. A section $f_{0}$ transverse off 0 yields a pair $X_{0} \subset X \subset \mathbb{C}^{n}$, where $X, 0$ is an ICIS, and $X_{0}$ is the intersection of an almost free divisor with $X$. The $\mathcal{K}_{\mathbb{C}^{p_{1}}, V_{1}}$-discriminant of a versal unfolding $f$ consists of parameter values $u$ where either $X_{u}=f_{u 2}^{-1}(0)$ is not smooth or $f_{u 1} \mid X: X \rightarrow \mathbb{C}^{p_{1}}$ is not (algebraically) transverse to $V_{1}$.

To investigate the properties of the $\mathcal{K}_{V_{2}, V_{1}}$-discriminant for a pair $\left(V_{2}, V_{1}\right)$, we introduce the subgroup

$$
\mathcal{K}_{V_{2}, V_{1}}^{*}=\mathcal{K}_{V_{2}, V_{1}} \cap \mathcal{K}_{V_{2}}^{*}
$$

where the group $\mathcal{K}_{V_{2}}^{*}$ is the CM-reduction of $\mathcal{K}_{V_{2}}$ (recall $\S 1$ and see Part II, [D6, $\S 6])$. We shall see in $\S 4$ that this is again a geometric subgroup of $\mathcal{A}$ or $\mathcal{K}$.

Theorem 5. For the free complete intersection pair $\left(V_{2}, V_{1}\right)$, suppose $n<h n\left(V_{1}\right)$, $h\left(V_{2}\right)$. Then, for nonlinear sections $f_{0}: \mathbb{C}^{n}, 0 \rightarrow \mathbb{C}^{p}, 0, \mathcal{K}_{V_{2}, V_{1}}^{*}$ is a Cohen-Macaulay reduction for $\mathcal{K}_{V_{2}, V_{1}}$. Hence, for a $\mathcal{K}_{V_{2}, V_{1}}$-versal unfolding $F$ of $f_{0}$, the $\mathcal{K}_{V_{2}, V_{1}}$ discriminant $D_{V_{2}, V_{1}}(F)$ is a free* divisor defined by

$$
\operatorname{Derlog}^{*}\left(D_{V_{2}, V_{1}}(F)\right)=\text { module of } \mathcal{K}_{V_{2}, V_{1}}^{*} \text {-liftable vector fields. }
$$

In the special case of a pair $\left(\mathbb{C}^{p_{1}}, V_{1}\right)$, where $V_{1}$ generically has Morse-type singularities in appropriate dimensions, this free* structure is reduced and $D_{V_{2}, V_{1}}(F)$ is a free divisor.

Theorem 6. Consider a complete intersection pair $V_{1}, 0 \subset \mathbb{C}^{p_{1}}, 0 \subset \mathbb{C}^{p}, 0$, so $V_{1}, 0 \subset \mathbb{C}^{p_{1}}, 0$ a free divisor. Let $p=p_{1}+p_{2}$. Suppose $f_{0}: \mathbb{C}^{n}, 0 \rightarrow \mathbb{C}^{p}, 0$ is a nonlinear section transverse to $\left(\mathbb{C}^{p_{1}}, V_{1}\right)$ off 0 with $n>p_{2}$, so that $X=f_{0}^{-1}\left(\mathbb{C}^{p_{1}}\right)$ is an 
ICIS of dimension $n-p_{2}>0$. Provided $V_{1}$ generically has Morse-type singularities in dimension $\operatorname{dim}(X)$ and $\operatorname{dim}(X)<h n\left(V_{1}\right)$, then the $\mathcal{K}_{\mathbb{C}^{p_{1}}, V_{1}}-$ discriminant $D_{\mathbb{C}^{p_{1}}, V_{1}}(F)$ of the versal unfolding $F$ of $f_{0}$ is a free divisor. Moreover,

$$
\operatorname{Derlog}\left(D_{\mathbb{C}^{p_{1}}, V_{1}}(F)\right)=\text { module of } \mathcal{K}_{\mathbb{C}^{p_{1}}, V_{1}}^{*} \text {-liftable vector fields. }
$$

Remark . As used in Theorem $2, h n\left(V_{1}\right)$ refers instead to codimension in $\mathbb{C}^{p_{1}}$.

The proofs of these theorems will be given in $\S 4$ and $\S 5$.

Applications to Relative Complete Intersections. We consider $\left(X, X_{0}\right)$, which consist of an ICIS $X, 0 \subset \mathbb{C}^{n}, 0$ of codimension $p_{2}$ and $X_{0}$ which is the intersection, transverse off 0 , of $X$ and an almost free divisor $V_{1}^{\prime}, 0$ obtained by pullback of the free divisor $V_{1}, 0 \subset \mathbb{C}^{p_{1}}, 0$. The versal unfolding allows both the almost free divisor $V_{1}^{\prime}$ and the isolated complete intersection $X$ to vary with the parameters $u$, and the equivalence captures the ambient equivalence of the pair $X_{0 u}=V_{1}^{\prime}{ }_{u} \cap X_{u} \subset X_{u}$. The relative discriminant $D_{\mathbb{C}^{p_{1}}, V_{1}}(F)$ of the versal unfolding $F$ consists of parameter values $u$ where either $X_{u}$ is not smooth, or $X_{u}$ is smooth but not transverse to $V_{1}^{\prime}$, i.e. the restriction $g_{u} \mid X_{u}$ is not transverse to $V_{1}$.

\section{(3.3) Isolated Hypersurface Singularities on ICIS}

We consider $g_{0}: X, 0 \rightarrow \mathbb{C}, 0$ which has an isolated singularity so that $X_{0}=$ $X \cap g^{-1}(0)$ is again an ICIS. Then, $\mathcal{K}_{\mathbb{C},\{0\}}$-equivalence for $\left(g_{0}, f_{0}\right)$ is equivalent to considering the $\mathcal{K}$-equivalence of $g_{0} \mid X$, where both $X$ and $g_{0}$ are allowed to vary. In this case, $V_{1}=\{0\} \subset \mathbb{C}$, so the genericity of Morse-type singularities trivially holds. Morse-type singularities consist of $g_{0} \mid X, x_{0}$ where $\left(X, x_{0}\right)$ is a smooth germ and $g_{0} \mid X$ has a standard Morse singularity at $x_{0}$. Now the relative discriminant corresponds to the discriminant for the versal deformation of $g_{0} \mid X$. The geometric description just given of the discriminant for a versal unfolding $F=\left(G, F_{1}\right)$ becomes: either the fiber $X_{u}$ is not smooth or it is smooth but $\bar{G}(\cdot, u) \mid X_{u}$ has a singularity. By Theorem 6 , we conclude the discriminant of the versal unfolding is a free divisor.

We mention that the recent results of Goryunov [Go2], and Mond-Van Straten [MVS] deduce the freeness of the discriminant for functions on space curves (in $\mathbb{C}^{3}$ ) from a " $\mu=\tau$ " result. For complete intersection curves in $\mathbb{C}^{n}$, we obtain the freeness from Theorem 6 but for $\mathcal{K}$-equivalence of the function on the deforming curve. The Mond-Van Straten " $\mu=\tau$ "result suggests that this should be the same as $\mathcal{R}$-equivalence of the function on the deforming curve.

\section{(3.4) Generic Hyperplane Arrangements on an ICIS}

Second, we consider the Boolean hyperplane arrangement $A_{p} \subset \mathbb{C}^{p}$, which consists of the coordinate hyperplanes of $\mathbb{C}^{p}$ and satisfies $h n\left(A_{p}\right)=\infty$. For a linear embedding germ $g_{0}: \mathbb{C}^{n}, 0 \rightarrow \mathbb{C}^{p}, 0$ which is transverse to (the strata of ) $A_{p}$ off 0 , $A=g_{0}^{-1}\left(A_{p}\right)$ is a generic arrangement of hyperplanes. Suppose $A$ is transverse to the ICIC $X$ off 0 . The versal deformation of $A^{\prime}=A \cap X$, allowing both $g_{0}$ and $X$ to deform, has discriminant the $\mathcal{K}_{\mathbb{C}^{p}, A_{p}}$-discriminant. By Theorem 6 , this is always a free divisor.

(3.5) Generic Arrangement of Isolated Hypersurface Singularities on ICIS 
More generally we can replace the linear embedding in 2) by any germ $g_{0}$ : $\mathbb{C}^{n}, 0 \rightarrow \mathbb{C}^{p}, 0$ which is transverse to $A_{p}$ off $0, V^{\prime}=g_{0}^{-1}\left(A_{p}\right)$ is now a generic nonlinear arrangement of hypersurfaces with isolated singularities at 0 . For a deformation $g_{u}$ of $g_{0}$ and $X_{u}$ of $X$, a stable point corresponds to a pair $X_{u}, A_{u}$ where $X_{u}$ is smooth and $A_{u}=g_{u}^{-1}\left(A_{p}\right) \cap X_{u}$ is a normal crossing divisor on $X_{u}$. The discriminant corresponds to values $u$ where this fails. If $V^{\prime}$ is algebraically transverse to $X$ off 0 , then again the versal deformation of $X_{0}=V^{\prime} \cap X$, allowing both $g_{0}$ and $X$ to deform, has discriminant the $\mathcal{K}_{\mathbb{C}^{p}, A_{p}}$-discriminant which again by Theorem 6 is always a free divisor.

\section{(3.6) Almost Free Hypersurface Arrangements on ICIS}

Suppose in either (3.4) or (3.5), we replace the Boolean arrangement $A_{p}$ by any other free hyperplane arrangement $A$. Then, $h n(A)=\infty$, although $A$ does not generically have Morse-type singularities. We may still conclude by Theorem 1 that the $\mathcal{K}_{\mathbb{C}^{p}, A^{-} \text {-discriminant is a free* divisor. }}$

\section{(3.7) ICIS Intersecting a Discriminant}

Suppose instead that we consider the composition of mappings

$$
\mathbb{C}^{m}, 0 \stackrel{f_{1}}{\longrightarrow} \mathbb{C}^{n}, 0 \stackrel{f_{0}}{\longrightarrow} \mathbb{C}^{p}, 0
$$

where $m \geq n>p, f_{1}$ is a finite $\mathcal{A}$-codimension germ with discriminant $D\left(f_{1}\right)$, and $f_{0}$ defines an ICIS $X$. We consider the behavior of the pair $X_{0}=D\left(f_{1}\right) \cap X \subset X$ where we allow both $f_{1}$ and $f_{0}$ to vary.

If $F_{1}: \mathbb{C}^{m^{\prime}}, 0 \rightarrow \mathbb{C}^{n^{\prime}}, 0$ denotes the stable unfolding of $f_{1}$, then $f_{1}$ is induced by pull-back by a germ $g_{1}: \mathbb{C}^{n}, 0 \rightarrow \mathbb{C}^{n^{\prime}}, 0$ and $D\left(f_{1}\right)=g_{1}^{-1}\left(D\left(F_{1}\right)\right)$. Then, the versal deformation of $X_{0} \subset X$ allowing both $f_{1}$ and $f_{0}$ to vary has discriminant which is the $\mathcal{K}_{\mathbb{C}^{n^{\prime}}, D\left(F_{1}\right)}$-discriminant. By Theorem 5 , provided $n-p<h n\left(D\left(F_{1}\right)\right)$, this discriminant is a free* divisor. The value of $h n\left(D\left(F_{1}\right)\right)$ is given by the results in $\S 6$ of part I for various classes of germs $F_{1}$.

Moreover, Theorem 6 also implies that when $f_{1}$ belongs to the "distinguished bifurcation class" in Part I, $\S 6$, then the $\mathcal{K}_{\mathbb{C}^{n^{\prime}}, D\left(F_{1}\right)}$-discriminant is a free divisor.

Mappings on Fixed Free Complete Intersections. Next, suppose $V, 0$ is fixed, and consider map germs on $V$. We suppose $V, 0 \subset \mathbb{C}^{n}, 0$ is a free complete intersection with good defining equation $h$. We consider germs $f_{0} \mid V: V, 0 \rightarrow \mathbb{C}^{m}, 0$ where $f_{0}: \mathbb{C}^{n}, 0 \rightarrow \mathbb{C}^{m}, 0$ defines an ICIS, under $\mathcal{A}(V), \mathcal{K}(V)$, or $\mathcal{R}^{+}(V)$-equivalence (where $m=1$ for $\mathcal{R}^{+}(V)$ ) as in Table 1 .

For $\mathcal{K}(V)$-equivalence, the $\mathcal{K}(V)$-discriminant for a versal unfolding $f(x, u)=$ $\left(f_{u}(x), u\right)$ of $f_{0}$ can be described geometrically as follows. We let $X_{u}=f_{u}^{-1}(0)$.

$$
\begin{array}{r}
D_{\mathcal{K}(V)}(F)=\left\{u \in \mathbb{C}^{q}: \text { there is an } x \in V \text { so either } X_{u} \text { is not smooth at } \mathrm{x}\right. \\
\text { or is not algebraically transverse to } V \text { at } \mathrm{x}\}
\end{array}
$$

To deduce properties of the $\mathcal{K}(V)$-discriminant, we introduce the subgroup $\mathcal{K}(V)^{*}$ for which the diffeomorphisms in the source are restrictions to $V$ of diffeomorphisms which preserve the fibers of $h$.

Theorem 7. Suppose that $V$ is a free complete intersection and that $f_{0}: \mathbb{C}^{n}, 0 \rightarrow$ $\mathbb{C}^{m}, 0, n \geq m$, has finite $\mathcal{K}(V)$-codimension with versal unfolding $F$. If $n-m<$ $h(V)$, then $\mathcal{K}(V)^{*}$ is a Cohen-Macaulay reduction of $\mathcal{K}(V)$, so the discriminant 
$D_{\mathcal{K}(V)}(F)$ is a free* divisor defined by

$\operatorname{Derlog}^{*}\left(D_{\mathcal{K}(V)}(F)\right)=$ module of $\mathcal{K}(V)^{*}$-liftable vector fields.

Remark . Even if $V$ generically has Morse-type singularities, we cannot improve the conclusion of the theorem because even for Morse-type singularities the appropriate vector fields are not, in general, $\mathcal{K}(V)^{*}$-liftable. We encountered this problem in part II for $\mathcal{K}_{V}$ when $V$ is a free complete intersection (recall [D6, Prop. 8.1]. Now the problem persists even for $V$ a free divisor.

As a result of the theorem, we obtain a result for $\mathcal{A}(V)$-equivalence, using the relation between $\mathcal{K}(V)$-versal unfoldings and $\mathcal{A}(V)$-stable germs (analogous to that for $\mathcal{K}$ and $\mathcal{A}$ due to Mather and Martinet as described earlier). Hence, an analogue of Theorem 3 carries over to the $\mathcal{A}(V)$-discriminant of an $\mathcal{A}(V)$-stable map germs. The $\mathcal{A}(V)$-discriminant of a germ $f_{0}: \mathbb{C}^{n}, 0 \rightarrow \mathbb{C}^{m}, 0$ is defined to be

$$
\begin{array}{r}
D_{\mathcal{A}(V)}\left(f_{0}\right)=\left\{y \in \mathbb{C}^{m}: \text { there is an } x \in f_{0}^{-1}(y) \cap V\right. \text { such that } \\
\left.\qquad d f_{0}(x)\left(T_{\text {log }}(V)_{(x)}\right) \neq T_{y} \mathbb{C}^{m}\right\}
\end{array}
$$

Theorem 8. Suppose that $V, 0 \subset \mathbb{C}^{n}, 0$ is a free complete intersection and that $f_{0}: \mathbb{C}^{n}, 0 \rightarrow \mathbb{C}^{m}, 0, n \geq m$, is $\mathcal{A}(V)$-stable. Provided $n-m<h(V)$, the $\mathcal{A}(V)$ discriminant $D_{\mathcal{A}(V)}\left(f_{0}\right)$ is a free* divisor defined by

$\operatorname{Derlog}^{*}\left(D_{\mathcal{A}(V)}\left(f_{0}\right)\right)=$ module of $\mathcal{K}(V)^{*}$-liftable vector fields

There is a corresponding result for $\mathcal{R}^{+}(V)$ equivalence for germs $f_{0}: \mathbb{C}^{n}, 0 \rightarrow$ $\mathbb{C}, 0$. The versal unfolding $F(x, u)=\left(F_{u}(x), u\right): \mathbb{C}^{n+q}, 0 \rightarrow \mathbb{C}^{1+q}, 0$ of a germ $f_{0}$ under $\mathcal{R}^{+}(V)$-equivalence is also versal for $\mathcal{A}(V)$-equivalence. For $\mathcal{R}^{+}(V)$, the discriminant of $F$ viewed as a map germ is

$$
\begin{aligned}
\left\{(y, u) \in \mathbb{C}^{1+q}: \text { there is an } x\right. & \in V, \text { such that at } x \text { either } F_{u}^{-1}(y) \\
& \text { is not smooth or it is not transverse to } V\}
\end{aligned}
$$

Thus, we obtain as a corollary.

Corollary 9. Suppose that $V$ is a free complete intersection. Let $f_{0}: \mathbb{C}^{n}, 0 \rightarrow \mathbb{C}, 0$ have finite $\mathcal{R}^{+}(V)$-codimension with versal unfolding $F$. If $n-1<h(V)$, then the $\mathcal{R}^{+}(V)$-discriminant of the map germ $F$ is a free* divisor defined by the module of $\mathcal{K}(V)^{*}$-liftable vector fields.

The proofs of Theorems 7 and 8 and Corollary 9 will be given in $\S 8$.

In the absence of genericity of Morse type singularities, the module of $\mathcal{K}(V)$ liftable vector fields does not define a free divisor structure on the $\mathcal{K}(V)$-discriminant.

Example 3.3. We reconsider Example 2.2, except for $\mathcal{K}(V)$-equivalence. For $\mathcal{K}(V)$-equivalence, we have the same versal unfolding $f\left(x, u_{1}, u_{2}\right)=\left(x+u_{1} y+\right.$ $\left.u_{2}, u_{1}, u_{2}\right)$ with $\mathcal{K}(V)$-discriminant the $u_{2}$-axis. Again, the vector field $\frac{\partial}{\partial u_{2}}$ is not $\mathcal{K}(V)$-liftable, although $u_{1} \frac{\partial}{\partial u_{2}}$ is.

(38) Functions on Discriminants

As in (3.7), except we can alternatively consider the restriction $f_{0} \mid D\left(f_{1}\right)$ under $\mathcal{K}\left(D\left(f_{1}\right)\right.$ )-equivalence where $f_{1}$ is a stable germ and we only allow $f_{0}$ to vary. Provided $n-p<h n\left(D\left(f_{1}\right)\right)$, then the $\mathcal{K}\left(D\left(f_{1}\right)\right)$-discriminants of the $\mathcal{K}\left(D\left(f_{1}\right)\right)$ versal unfolding of $f_{0}$ is a free* divisor. 
Remark 3.4. For several examples with $V$ a free divisor, the $\mathcal{K}(V)$-discriminant is actually a free divisor. This raises the question of whether simple conditions will actually ensure that $N \mathcal{K}(V)_{u n, e} \cdot F$ is a Cohen-Macaulay $\mathcal{O}_{\mathbb{C}^{q}, 0}$-module. Then, provided $V, 0$ generically has Morse-type singularities in dimension $n$, we would have the genericity of $\mathcal{K}(V)$-liftable vector fields, yielding the freeness of $\mathcal{K}(V)$ discriminants.

\section{CM-Reduction for $\mathcal{K}_{V_{2}, V_{1}}$-EQuivalence}

In this section we prove Theorem 5 by showing that the relative $\mathcal{K}_{V_{2}, V_{1}}$-equivalence for pairs has a CM-reduction $\mathcal{K}_{V_{2}, V_{1}}^{*}$. The arguments share similarities to those given for $\mathcal{K}_{V}$-equivalence in $\S 6$ of Part II, so in places we merely outline certain steps.

To define the associated extended tangent spaces to $\mathcal{K}_{V_{2}, V_{1}}$ and its unfolding group $\mathcal{K}_{V_{2}, V_{1} \text { un }}$, we define analogously to $\operatorname{Derlog}(V)$

$$
\operatorname{Derlog}\left(V_{2}, V_{1}\right)=\left\{\zeta \in \theta_{p}: \zeta\left(I\left(V_{i}\right)\right) \subset I\left(V_{i}\right), i=1,2\right\} .
$$

Then, $\operatorname{Derlog}\left(V_{2}, V_{1}\right)=\operatorname{Derlog}\left(V_{1}\right) \cap \operatorname{Derlog}\left(V_{2}\right)$ is a finitely generated $\mathcal{O}_{\mathbb{C}^{p}, 0^{-}}$ module and the associated sheaf is also coherent. Let $\left\{\zeta_{1}, \ldots, \zeta_{r}\right\}$ be a set of generators. Then, the extended tangent spaces are given by

$$
T \mathcal{K}_{V_{2}, V_{1}, e} \cdot f_{0}=\mathcal{O}_{\mathbb{C}^{n}, 0}\left\{\frac{\partial f_{0}}{\partial x_{1}}, \ldots, \frac{\partial f_{0}}{\partial x_{n}}, \zeta_{1} \circ f_{0}, \ldots, \zeta_{r} \circ f_{0}\right\}
$$

and for an unfolding $F(x, u)=(\bar{F}(x, u), u)$,

$$
T \mathcal{K}_{V_{2}, V_{1}, u n, e} \cdot F=\mathcal{O}_{\mathbb{C}^{n+q}, 0}\left\{\frac{\partial \bar{F}}{\partial x_{1}}, \ldots, \frac{\partial \bar{F}}{\partial x_{n}}, \zeta_{1} \circ \bar{F}, \ldots, \zeta_{r} \circ \bar{F}\right\}
$$

We let $\left(V_{2}, V_{1}\right)$ denote a free complete intersection pair as in Definition 3.2 so $V_{2}=\mathbb{C}^{p_{1}} \times W_{2}$, and $V_{1}=W_{1} \times W_{2}$ for a free complete intersection $W_{2}$ and free divisor $W_{1}$. Let $H_{2}: \mathbb{C}^{p_{2}}, 0 \rightarrow \mathbb{C}^{k}, 0$ denote the free defining equation for $W_{2}$, and let $p=p_{1}+p_{2}$. If $\left\{\zeta_{i}^{(j)}\right\}$ is a set of generators for $\operatorname{Derlog}\left(W_{j}\right)$, and $H_{2}=\left(h_{1}, \ldots, h_{k}\right)$ defines $W_{2}$, then applying [D6, Prop. 5.6] we obtain

$$
\operatorname{Derlog}\left(V_{2}, V_{1}\right)=\mathcal{O}_{\mathbb{C}^{p}, 0}\left\{\zeta_{i}^{(1)} ; \zeta_{j}^{(2)} ; h_{\ell} \frac{\partial}{\partial y_{m}^{(1)}}\right\}
$$

where $i=1, \ldots, p_{1}, j=1, \ldots, r, \ell=1, \ldots, k$, and $m=1, \ldots, p_{1}$.

We then define the group

$$
\mathcal{K}_{V_{2}, V_{1}}^{*}=\mathcal{K}_{V_{2}, V_{1}} \cap \mathcal{K}_{V_{2}}^{*}
$$

Here $\mathcal{K}_{V_{2}}^{*}$ is the subgroup of $\mathcal{K}_{V_{2}}$ consisting of germs of diffeomorphisms of $\mathbb{C}^{p} \times \mathbb{C}^{n}, 0$ which restricted to $V_{2} \times \mathbb{C}^{n}$ are the restrictions of diffeomorphisms preserving the fibers of $H_{2} \circ \pi$ for $\pi$ the projection onto $\mathbb{C}^{p}$ (see $[\mathrm{D} 6, \S 6]$ ). $\mathcal{K}_{V_{2}, V_{1}}^{*}$ can be seen to be a geometric subgroup of $\mathcal{A}$ or $\mathcal{K}$.

To compute the extended tangent spaces, we let $\left\{\zeta_{1}, \ldots, \zeta_{p-k}\right\}$ denote $\left\{\zeta_{1}^{(1)}, \ldots\right.$, $\left.\zeta_{p_{1}}^{(1)}, \zeta_{1}^{(2)}, \ldots, \zeta_{p_{2}-k}^{(2)}\right\}$, which is the union of the sets of generators of $\operatorname{Derlog}\left(W_{1}\right)$ and $\operatorname{Derlog}\left(H_{2}\right)$. Then, we obtain for $f_{0}$ the extended tangent spaces

$$
T \mathcal{K}_{V_{2}, V_{1}, e}^{*} \cdot f_{0}=\mathcal{O}_{\mathbb{C}^{n}, 0}\left\{\frac{\partial f_{0}}{\partial x_{1}}, \ldots, \frac{\partial f_{0}}{\partial x_{n}}, \zeta_{1} \circ f_{0}, \ldots, \zeta_{p-k} \circ f_{0}\right\}+I\left(V_{2}\right) \cdot \theta\left(f_{0}\right)
$$


and

$T \mathcal{K}_{V_{2}, V_{1}, u n, e}^{*} \cdot F=\mathcal{O}_{\mathbb{C}^{n+q}, 0}\left\{\frac{\partial \bar{F}}{\partial x_{1}}, \ldots, \frac{\partial \bar{F}}{\partial x_{n}}, \zeta_{1} \circ \bar{F}, \ldots, \zeta_{p-k} \circ \bar{F}\right\}+I\left(V_{2}\right) \cdot \theta(\bar{F})$

We denote the sheaves of $\mathcal{O}_{\mathbb{C}^{n+q}, 0}$-modules associated to each of the normal spaces by $\mathcal{N} \mathcal{K}_{V_{2}, V_{1}, u n, e} \cdot F$ and $\mathcal{N} \mathcal{K}_{V_{2}, V_{1}, u n, e}^{*} \cdot F$.

Proposition 4.1. For the free complete intersection pair $\left(V_{2}, V_{1}\right)$ :

(1) If $n<h\left(V_{2}\right)$, then, $f_{0}: \mathbb{C}^{n}, 0 \rightarrow \mathbb{C}^{p}, 0$ has finite $\mathcal{K}_{V_{2}, V_{1}}$-codimension iff it has finite $\mathcal{K}_{V_{2}, V_{1}}^{*}$-codimension.

(2) For an unfolding $F$ of $f_{0}$,

$$
\operatorname{supp}\left(\mathcal{N} \mathcal{K}_{V_{2}, V_{1}, \text { un,e }} \cdot F\right)=\operatorname{supp}\left(\mathcal{N K}_{V_{2}, V_{1}, \text { un,e }}^{*} \cdot F\right)
$$

Proof of proposition 4.1. First, $f_{0}$ having finite $\mathcal{K}_{V_{2}, V_{1}}$-codimension is equivalent to $\operatorname{supp}\left(\mathcal{N K}_{V_{2}, V_{1}, e} \cdot f_{0}\right)=\{0\}$. An analogous result holds for $\mathcal{K}_{V_{2}, V_{1}}^{*}$. Thus, 1$)$ follows from 2) applied to $f_{0}$ as an unfolding on 0 parameters.

Since

$$
\mathcal{T} \mathcal{K}_{V_{1}, V_{2}, \text { un }, e}^{*} \subseteq \mathcal{T} \mathcal{K}_{V_{1}, V_{2}, \text { un,e }}
$$

there is the inclusion $\subseteq$ in (4.4). For the reverse inclusion, we verify that both sets are defined by the failure of geometric transversality to the pair.

For example, consider $\operatorname{supp}\left(\mathcal{N} \mathcal{K}_{V_{2}, V_{1}, u n, e}^{*} \cdot F\right)$. Because of the sheaf inclusion

$$
\mathcal{I}\left(V_{2}\right) \cdot \Theta(\bar{F}) \subset \mathcal{T} \mathcal{K}_{V_{2}, V_{1}, u n, e}^{*} \cdot F
$$

a point $(x, u) \in \operatorname{supp}\left(\mathcal{N} \mathcal{K}_{V_{2}, V_{1}, u n, e}^{*} \cdot F\right)$ implies $\bar{F}(x, u) \in V_{2}=\mathbb{C}^{p_{1}} \times W_{2}$.

If $\bar{F}(x, u)=y \in V_{2}$, then $(x, u) \notin \operatorname{supp}\left(\mathcal{N K}_{V_{2}, V_{1}, u n, e}^{*} \cdot F\right)$ is equivalent to

$$
T_{l o g}\left(W_{1}\right)_{(y)}+T_{l o g}\left(H_{2}\right)_{(y)}+D \bar{F}(x, u)\left(T_{x} \mathbb{C}^{n}\right)=T_{y} \mathbb{C}^{p}
$$

However, the assumption $n<h\left(V_{2}\right)$ implies (see [D2, §2]) that

$$
T_{l o g} H_{2(x)}=T_{l o g} W_{2(x)}=T_{x} S_{2}
$$

where $S_{2}$ denotes the canonical Whitney stratum of $W_{2}$ with $\bar{F}(x, u) \in \mathbb{C}^{p_{1}} \times S_{2}$. Then, using (4.6), we see (4.5) is equivalent to

$$
T_{\text {log }}\left(W_{1}\right)_{(y)}+T_{\text {log }}\left(W_{2}\right)_{(y)}+D \bar{F}(x, u)\left(T_{x} \mathbb{C}^{n}\right)=T_{y} \mathbb{C}^{p}
$$

First, suppose $y=\left(y_{1}, y_{2}\right) \in V_{1}$ with $y_{2} \in S_{2}$. We apply the argument used in Proposition 5.11 of Part II [D6] (except here $W_{2}$ is a free complete intersection rather than free divisor so we replace $h_{2}$ there by $\left.h_{2}, \ldots, h_{k}\right)$ to obtain

$$
T_{l o g}\left(W_{1}\right)_{\left(y_{1}\right)} \oplus T_{l o g}\left(W_{2}\right)_{\left(y_{2}\right)} \subseteq T_{l o g}\left(V_{1}\right)_{(y)} \subseteq T_{l o g}\left(W_{1}\right)_{\left(y_{1}\right)} \oplus T_{y_{2}} S_{2}
$$

By (4.6) we have equality in (4.8) so (4.7) becomes

$$
T_{\text {log }}\left(V_{1}\right)_{(y)}+D \bar{F}(x, u)\left(T_{x} \mathbb{C}^{n}\right)=T_{y} \mathbb{C}^{p}
$$

Hence, $\bar{F}(\cdot, u)$ is transverse to $V_{1}$ (and hence $V_{2}$ ) at $y$.

If instead $y \in V_{2} \backslash V_{1}$, then $T_{\log }\left(W_{1}\right)_{(y)}=T_{y} \mathbb{C}^{p_{1}}$ and

$$
T_{l o g}\left(V_{2}\right)_{(y)}=T_{y} \mathbb{C}^{p_{1}} \oplus T_{l o g}\left(W_{2}\right)_{(y)}
$$

Thus, (4.5) is equivalent to

$$
T_{\text {log }}\left(V_{2}\right)_{(y)}+D \bar{F}(x, u)\left(T_{x} \mathbb{C}^{n}\right)=T_{y} \mathbb{C}^{p}
$$

Hence, $\bar{F}(\cdot, u)$ is algebraically transverse to $V_{2}$ at $(x, u)$ (and trivially to $V_{1}$ ). 
We conclude $(x, u) \notin \operatorname{supp}\left(\mathcal{N} \mathcal{K}_{V_{2}, V_{1}, u n, e}^{*} \cdot F\right)$ is equivalent to $\bar{F}(\cdot, u)$ being algebraically transverse to both $V_{1}$, and $V_{2}$.

Similar arguments establish that $\operatorname{supp}\left(\mathcal{N K}_{V_{2}, V_{1}, u n, e} \cdot F\right)$ consists of points $(x, u)$ where $\bar{F}(\cdot, u)$ fails to be algebraically transverse to both $V_{2}$ and $V_{1}$.

Then, we define the critical set in terms of these normal sheaves.

Definition 4.2. We define the $\mathcal{K}_{V_{2}, V_{1}}$-critical set of $F$ to be

$$
C_{V_{2}, V_{1}}(F)=\operatorname{supp}\left(\mathcal{N} \mathcal{K}_{V_{2}, V_{1}, \text { un,e }} \cdot F\right) \text {. }
$$

and analogously for the $\mathcal{K}_{V_{2}, V_{1}}^{*}$ critical set

Suppose $f_{0}$ has finite $\mathcal{K}_{V_{2}, V_{1}}$-codimension and $n<h\left(V_{2}\right)$. By proposition 4.1, if $F$ is an unfolding of $f_{0}$, the critical sets $C_{V_{2}, V_{1}}(F)$ for $\mathcal{K}_{V_{2}, V_{1}}$ and $C_{V_{2}, V_{1}}^{*}(F)$ for $\mathcal{K}_{V_{2}, V_{1}}^{*}$ agree and $\pi \mid C_{V_{2}, V_{1}}(F)$ is finite to one. By Grauert's theorem, $\mathcal{N} \mathcal{K}_{V_{2}, V_{1}} \cdot F \stackrel{\text { def }}{=}$ $\pi_{*}\left(\mathcal{N} \mathcal{K}_{V_{2}, V_{1}, \text { un }, e} \cdot F\right)$ and $\mathcal{N} \mathcal{K}_{V_{2}, V_{1}}^{*} \cdot F \stackrel{\text { def }}{=} \pi_{*}\left(\mathcal{N} \mathcal{K}_{V_{2}, V_{1}, \text { un,e }}^{*} \cdot F\right)$ are coherent and are the sheaves associated to the normal spaces for each group. Hence, by Proposition 4.1 they have common support the $\mathcal{K}_{V_{2}, V_{1}}$ and $\mathcal{K}_{V_{2}, V_{1}}^{*}$-discriminants of $F$. We conclude that the $\mathcal{K}_{V_{2}, V_{1}}$ and $\mathcal{K}_{V_{2}, V_{1}}^{*}$ critical sets and discriminants of $F$ are analytic subsets of the same dimensions.

The proof of Theorem 1 is completed by proving the following proposition and applying Theorem 2 of part II [D6].

Proposition 4.3. Let $\left(V_{2}, V_{1}\right)$ be a free complete intersection pair. Suppose that $f_{0}: \mathbb{C}^{n}, 0 \rightarrow \mathbb{C}^{p}, 0$ has finite $\mathcal{K}_{V_{2}, V_{1}}$-codimension with $k \leq n<h n\left(V_{1}\right), h\left(V_{2}\right)$. Let $F: \mathbb{C}^{n+q}, 0 \rightarrow \mathbb{C}^{p+q}, 0$ be a $\mathcal{K}_{V_{2}, V_{1}}$-versal unfolding of $f_{0}$. Then, $\mathcal{N K}_{V_{2}, V_{1}}^{*} \cdot F$ is Cohen-Macaulay with $\operatorname{supp}\left(\mathcal{N} \mathcal{K}_{V_{2}, V_{1}}^{*} \cdot F\right)=D_{V_{2}, V_{1}}^{*}(F)$ of dimension $q-1$.

Remark . We require $k \leq n$ so that $f_{0}^{-1}\left(V_{2}\right)$ is a complete intersection.

Proof. Dividing both sides of $(4.3)$ by $I\left(V_{2}\right) \cdot \theta(\bar{F})$ we obtain

$$
\begin{aligned}
N \mathcal{K}_{V_{2}, V_{1}, u n, e}^{*} \cdot F \simeq \\
\quad \mathcal{O}_{\mathcal{X}, 0}^{p} / \mathcal{O}_{\mathcal{X}, 0}\left\{\frac{\partial \bar{F}}{\partial x_{1}}(x, u), \ldots, \frac{\partial \bar{F}}{\partial x_{n}}(x, u), \zeta_{1} \circ \bar{F}(x, u), \ldots, \zeta_{p-k} \circ \bar{F}(x, u)\right\}
\end{aligned}
$$

By proposition 4.1 , the $\mathcal{O}_{\mathcal{X}, 0}$-module $N \mathcal{K}_{V_{2}, V_{1}, \text { un,e }}^{*} \cdot F$ has support $C_{V_{2}, V_{1}}^{*}(F)=$ $C_{V_{2}, V_{1}}(F)$.

We then apply the argument similar to that given in proposition 6.12 of Part

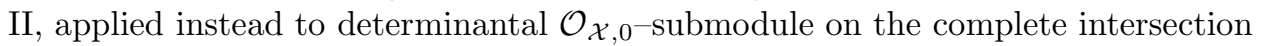
$\mathcal{X}=\bar{F}^{-1}\left(V_{2}\right)$ of codimension $k$. As $N \mathcal{K}_{V_{2}, V_{1}, u n, e}^{*} \cdot F$ is a quotient of $\mathcal{O}_{\mathcal{X}, 0}^{p}$ by the $\mathcal{O}_{\mathcal{X}, 0}$-submodule generated by $n+p-k$ generators, we may apply results of EagonNorthcott [EN], to conclude $C_{V_{2}, V_{1}}^{*}(F)$ has codimension $\leq n+p-k-(p-1)=$ $n-k+1$ in $\mathcal{X}$ so it has dimension $\geq n+q-k-(n-k+1)=q-1$ with equality implying it is Cohen-Macaulay. As $\mathcal{N K}_{V_{2}, V_{1}}^{*} \cdot F$ is the sheaf associated to the push forward of the normal space $N \mathcal{K}_{V_{2}, V_{1}, u n, e}^{*} \cdot F$, the remainder of the proof for the equality of dimensions follows as in the proof of proposition 6.12 of Part II using again the parametrized transversality theorem and the geometric characterization of $D_{V_{2}, V_{1}}(F)$ using $\mathcal{K}_{V_{2}, V_{1}}$ to prove $\operatorname{dim} D_{V_{2}, V_{1}}^{*}(F)=\operatorname{dim} D_{V_{2}, V_{1}}(F)=q-1$. Then, both $\mathcal{N} \mathcal{K}_{V_{2}, V_{1}, \text { un }, e}^{*} \cdot F$ and $\mathcal{N K}_{V_{2}, V_{1}}^{*} \cdot F$ are Cohen-Macaulay as $\mathcal{O}_{\mathcal{X}, 0}$-modules and then so are the pushforwards as $\mathcal{O}_{\mathbb{C}^{q}, 0^{-}}$modules. 


\section{5. $\mathcal{K}_{\mathbb{C}^{p}, V^{-D i s c r i m i n a n t s ~ a S ~ F r e e ~ D i v i s o r s ~}}$}

We have already established that $\mathcal{K}_{\mathbb{C}^{p_{1}}, V_{1}}^{*}$ is a Cohen-Macaulay reduction of $\mathcal{K}_{\mathbb{C}^{p_{1}}, V_{1}}$ in $\S 4$. To prove Theorem 6 , we will apply Theorem 1 of Part II. It is sufficient to establish the next proposition.

Proposition 5.1. Let $V_{1}, 0 \subset \mathbb{C}^{p_{1}}, 0$ be a free divisor and let $p=p_{1}+p_{2}$. Suppose $0<n-p_{2}<h n\left(V_{1}\right)$ and that $V_{1}, 0$ has Morse-type singularities in dimension $n-p_{2}$. Then, versal unfoldings $F$ of germs $f_{0}: \mathbb{C}^{n}, 0 \rightarrow \mathbb{C}^{p}, 0$ have generically $\mathcal{K}_{\mathbb{C}^{p_{1}}, V_{1}}^{*}$-liftable vector fields.

To prove this proposition, we apply the criterion (1.3) to verify that $\mathcal{K}_{\mathbb{C}^{p_{1}}, V_{1}}$ generically has $\mathcal{K}_{\mathbb{C}^{p_{1}}, V_{1}}^{*}$-liftable vector fields for the pair $V_{1} \subset \mathbb{C}^{p_{1}} \subset \mathbb{C}^{p}$. we view $\mathbb{C}^{p_{1}}$ as stratified by $\mathbb{C}^{p_{1}} \backslash V_{1}$ and the canonical Whitney stratification of $V_{1}$.

First, there is a local form of $\mathcal{K}_{\mathbb{C}^{p_{1}}, V_{1}}^{*}$-equivalence in a neighborhood of each $y \in \mathbb{C}^{p_{1}}$, and the module of $\mathcal{K}_{\mathbb{C}^{p_{1}}, V_{1}}^{*}$-liftable vector fields restricts by coherence to the module of locally liftable vector fields for the local $\mathcal{K}_{\mathbb{C}^{p_{1}}, V_{1}}^{*}$-equivalence for all $y$ in a neighborhood of 0 . This establishes $(1)$ of $(1.3)$. Note we view $\mathcal{K}_{\mathbb{C}^{p_{1}}, V_{1}}^{*}$ as a subgroup of $\mathcal{K}_{\mathbb{C}^{p_{1}}}^{*}$, and in the case of $y \in \mathbb{C}^{p_{1}} \backslash V_{1}, \mathcal{K}_{\mathbb{C}^{p_{1}}}^{*}$ locally reduces to just $\mathcal{K}_{\mathbb{C}^{p_{1}}-\text { equivalence. }}$

Next, provided that $V_{1}$ generically has Morse-type singularities in dimension $n$, so does $\mathbb{C}^{p_{1}}$ as the remaining stratum $\mathbb{C}^{p_{1}} \backslash V_{1}$ has Morse-type singularities in dimension $n$ by Example 7.7 of Part II. This establishes (2) of (1.3).

Third, to establish condition 3) of (1.3), we must show that there is a Zariski open subset of jets which are algebraically transverse to $V_{1}$ and with respect to a local form of $\mathcal{K}_{\mathbb{C}^{p_{1}}, V_{1}}^{*}$-equivalence at the target point $y$, the jet has $\mathcal{K}_{\mathbb{C}^{p_{1}}, V_{1}, e^{- \text {codim }}}^{*}$ 0 . The verification in $\S 4$ that $\mathcal{K}_{\mathbb{C}^{p_{1}}, V_{1}}^{*}$ is a $\mathrm{CM}$-reduction implies that germs have

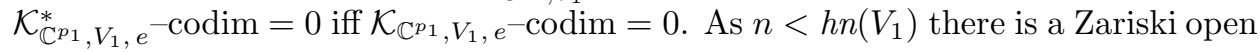
subset of jets of germs algebraically transverse to the stratified set $\mathbb{C}^{p_{1}}$ (consisting of $\mathbb{C}^{p_{1}}$ and the strata of $V_{1}$ ). Hence, they are also algebraically transverse to $V_{1}$,

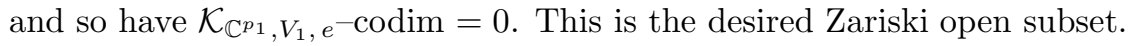

Finally, to establish the remaining condition (4) of (1.3) we must exhibit a Zariski open subset $\mathcal{W}$ of the set of jets which are not algebraically transverse to (the stratification of) $\mathbb{C}^{p_{1}}$ so that for each point $y$ in a stratum of $\mathbb{C}^{p_{1}}$ of codimension $\leq n+1$ : i) any $z \in \mathcal{W}$ with target $y$ is the jet of a Morse-type singularity and has

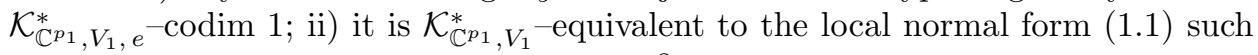
that for the versal unfolding $F$ in (1.2), $u \frac{\partial}{\partial u}$ is $\mathcal{K}_{\mathbb{C}^{p_{1}}, V_{1}}^{*}$-liftable.

If $y \in \mathbb{C}^{p_{1}} \backslash V_{1}$, then we generically have Morse-type singularities for $\mathbb{C}^{p_{1}}$ in all dimensions, which are then $\mathcal{K}_{\mathbb{C}^{p_{1}}, V_{1}}^{*}$ Morse-type singularities since the action of $\mathcal{K}_{\mathbb{C}^{p_{1}}, V_{1}}^{*}$ at points $y \in \mathbb{C}^{p_{1}} \backslash V_{1}$ is just $\mathcal{K}_{\mathbb{C}^{p_{1}}}^{*}$-equivalence. We then observe that $\mathcal{K}_{\mathbb{C}^{p_{1}}, \text { un }}^{*}=\mathcal{K}_{\mathbb{C}^{p_{1}}, \text { un }}$ because in this case of smooth $\mathbb{C}^{p_{1}}$, any $\mathcal{K}_{\mathbb{C}^{p_{1}}, \text { un }}$-equivalence restricted to $\mathbb{C}^{p_{1}}$ is also the restriction of a $\mathcal{K}_{H_{2}, u n}$-equivalence.

If instead $y \in V_{1}$, locally we reduce to the case of $y=0$ and consider $f_{0}$ a weighted homogeneous germ (for local coordinates such that $V_{1}$ is also weighted

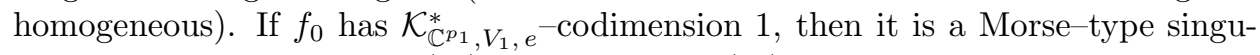

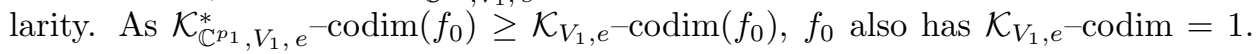
Otherwise, if $\mathcal{K}_{V_{1}, e}-\operatorname{codim}\left(f_{0}\right)=0$, then $f_{0}$ would be algebraically transverse to $V_{1}$ at 0 . Thus, using the definition of the $\mathcal{K}_{\mathbb{C}^{p_{1}}, V_{1}, e}^{*}$ normal space $(4.2), \mathcal{K}_{\mathbb{C}^{p_{1}}, V_{1}, e^{-}}^{*}$ $\operatorname{codim}\left(f_{0}\right)=0$, a contradiction. 
Next, we identify a Zariski open subset of the jets of Morse-type singularities which are $\mathcal{K}_{\mathbb{C}^{p_{1}}, V_{1}}^{*}$ Morse-type singularities.

Proposition 5.2. Let $V_{1}, 0 \subset \mathbb{C}^{p_{1}}, 0$ be a free divisor with $p=p_{1}+p_{2}$. For $f_{0}=\left(f_{01}, f_{02}\right): \mathbb{C}^{n}, 0 \rightarrow \mathbb{C}^{p}, 0$, suppose that $f_{01}$ is a Morse-type singularity for $V_{1}$ with $y_{p_{1}}$ the exceptional weight coordinate (in the sense of [D5, Def. 4.28]). Second, suppose that $d f_{02}(0): \operatorname{ker}\left(d f_{01}(0)\right) \rightarrow \mathbb{C}^{p_{2}}$ is onto. Then, up to $\mathcal{K}_{\mathbb{C}^{p_{1}}, V_{1}}^{*}$ equivalence, we may assume $V_{1}, 0=\mathbb{C}^{r} \times V_{0}, 0$ for $V_{0}, 0 \subset \mathbb{C}^{p_{1}^{\prime}}, 0$, and with respect to coordinates for which $V_{0}, 0$ is weighted homogeneous, $f_{0}$ has the form

$$
f_{0}\left(x_{1}, \ldots, x_{n}\right)=\left(0, \ldots, 0, x_{1}, \ldots, x_{p_{1}^{\prime}-1}, \sum_{i=p_{1}^{\prime}+p_{2}}^{n} x_{i}^{2}, x_{p_{1}^{\prime}}, \ldots, x_{p_{1}^{\prime}+p_{2}-1}\right)
$$

Example 5.3. In the case of $V=\{0\} \subset \mathbb{C}, f_{0}=\left(\sum_{i=1+p_{2}}^{n} x_{i}^{2}, x_{1}, \ldots, x_{p_{2}}\right)$, which states exactly that $X=f_{02}^{-1}(0)=\mathbb{C}^{n-p_{2}}$, and $f_{01} \mid X$ is a standard Morse singularity.

Proof. Just as for the proof in Parts I and II of the normal form of Morse-type singularities for $\mathcal{K}_{V^{-}}$equivalence, we may first reduce to the case where $T_{l o g} V_{1(0)}=0$ so $p_{1}^{\prime}=p_{1}$. Then, since $f_{01}$ is a Morse-type singularity for $V_{1}, 0 \subset \mathbb{C}^{p_{1}}, 0$, we may apply $\mathcal{K}_{V_{1}}$-equivalence to put $f_{01}$ into the normal form

$$
f_{01}\left(x_{1}, \ldots, x_{n}\right)=\left(x_{1}, \ldots, x_{p_{1}^{\prime}-1}, \varphi\left(x_{p_{1}^{\prime}}, \ldots, x_{n}\right)\right) \quad \text { where } \quad \varphi=\sum_{j=p_{1}^{\prime}}^{n} x_{j}^{2}
$$

$\mathcal{K}_{V_{1}}$ acting on $\mathbb{C}^{p_{1}}, 0$ may be extended to act trivially on $\{0\} \times \mathbb{C}^{p_{2}}$. Thus, we may view $\mathcal{K}_{V_{1}} \subset \mathcal{K}_{\mathbb{C}^{p_{1}}, V_{1}}^{*}$. Hence, both of these changes of coordinates are in $\mathcal{K}_{\mathbb{C}^{p_{1}}, V_{1}}^{*}$. Next, the condition that $d f_{02}(0): \operatorname{ker}\left(d f_{01}(0)\right) \rightarrow \mathbb{C}^{p_{2}}$ is onto is preserved by the $\mathcal{K}_{V_{1}}$-action; $f_{02}$ is composed with the same diffeomorphism on $\mathbb{C}^{n}$ as $f_{01}$. We still denote this germ by $f_{0}=\left(f_{01}, f_{02}\right)$. Next, by a linear change of coordinates on $\left(x_{p_{1}^{\prime}}, \ldots, x_{n}\right)$, we may assume

$$
d f_{02}(0)\left(x_{p_{1}^{\prime}}, \ldots, x_{n}\right)=\left(x_{p_{1}^{\prime}}, \ldots, x_{p_{1}^{\prime}+p_{2}-1}\right)
$$

Under this linear change, $\varphi$ has changed but it remains nondegenerate, and we still denote it by $\varphi$. Then, a straightforward computation using (4.18) of Part I shows that with $f_{02}$ satisfying (5.3)

$$
\begin{aligned}
T \mathcal{K}_{\mathbb{C}^{p_{1}}, V_{1}}^{*} \cdot f_{0}=m_{n}\{ & \left.\frac{\partial}{\partial y_{1}^{(1)}}, \ldots, \frac{\partial}{\partial y_{p-1}^{(1)}}\right\}+\left(x_{1}, \ldots, x_{p-1}\right)\left\{\frac{\partial}{\partial y_{p}}\right\} \\
& +m_{n}\left\{\frac{\partial}{\partial y_{1}^{(2)}}, \ldots, \frac{\partial}{\partial y_{p_{2}}^{(2)}}\right\}+m_{n}^{2}\left\{\frac{\partial}{\partial y_{p}}\right\}
\end{aligned}
$$

Then we can apply Mather's geometric lemma (i.e. in the form of the addendum of part I [D5]) to conclude that $f_{0}$ is $\mathcal{K}_{\mathbb{C}^{p_{1}}, V_{1}}^{*}$-equivalent to the normal form.

We now assert: i) the condition in Proposition 5.2 that $d f_{02}(0): \operatorname{ker}\left(d f_{01}(0)\right) \rightarrow$ $\mathbb{C}^{p_{2}}$ is onto defines a Zariski open subset $\mathcal{W}$ of the jets defining Morse-type singularities; ii) that such singularities in normal form (5.1) have $\mathcal{K}_{\mathbb{C}^{p_{1}}, V_{1}, e}^{*}-\operatorname{codim}=1$; and iii) the vector field $u \frac{\partial}{\partial u}$ is $\mathcal{K}_{\mathbb{C}^{p_{1}}, V_{1}}^{*}$-liftable. This will complete the claim in Proposition 5.1. 
A straighforward calculation shows that a germ $f_{0}$ which is a Morse-type sin-

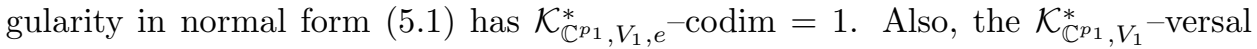
unfolding is given by

$$
F(x, u)=(\bar{F}(x, u), u) \quad \text { with } \quad \bar{F}(x, u)=f_{0}(x)+(0, \ldots, 0, u, 0, \ldots, 0)
$$

where $u$ is in the $p_{1}$-th coordinate. Then, iii) follows from the next Lemma.

Lemma 5.4. Suppose $f_{0}$ is a Morse-type singularity in normal form (5.1). If the exceptional weight of $f_{01}, w t\left(y_{p_{1}}\right)$, is not zero, then $u \frac{\partial}{\partial u}$ is $\mathcal{K}_{\mathbb{C}^{p_{1}}, V_{1}}^{{ }_{1}}$-liftable.

Proof. Because

$$
m_{p_{2}} \theta\left(f_{0}\right)+\operatorname{Derlog}\left(V_{1}\right) \subset T \mathcal{K}_{\mathbb{C}^{p_{1}}, V_{1}}^{*}
$$

it follows that $T \mathcal{K}_{\mathbb{C}^{p_{1}}, V_{1}}^{*}$ does contain the Euler vector field. Hence, liftability follows exactly as for Lemma 4.10 in part I [D5].

Finally, we establish i). Let $\Sigma_{1}$ denote the set 2-jets of germs which fail to be algebraically transverse to $V_{1}\left(=V_{1} \times\{0\}\right)$.

Lemma 5.5. If $V, 0 \subset \mathbb{C}^{p}, 0$ has $\mathcal{K}_{\mathbb{C}^{p_{1}}, V_{1}}$ Morse-type singularities, then $\mathcal{W}$ is a Zariski open dense subset of $\Sigma_{1}$ consisting of jets of $\mathcal{K}_{\mathbb{C}^{p_{1}}, V_{1}}^{*}$ Morse-type singularities.

Proof. The set $U_{1}$ of 2-jets of germs $f_{0}=\left(f_{01}, f_{02}\right)$ for which $f_{01}$ is a Morse type singularity for $V_{1}$ is Zariski open and dense by Corollary 4.24 in Part I [D5]. As there is at least one Morse-type singularity, $n \geq p-1$ so for $f_{0} \in U_{1}$, $\operatorname{dim} \operatorname{ker}\left(d f_{01}(0)\right)=$ $n-\left(p_{1}-1\right) \geq p_{2}$. Thus, the set of $f_{0} \in U_{1}$ for which $d f_{02}(0): \operatorname{ker}\left(d f_{01}(0)\right) \rightarrow \mathbb{C}^{p_{2}}$ is onto is a Zariski open dense subset of $\Sigma_{1}$.

We conclude this section by indicating why there cannot be an analogous result when $\mathbb{C}^{p_{1}}$ is replaced by another free complete intersection $W_{2}$. The problem already arises for $\mathcal{K}_{V}^{*}$. We consider the simplest complete intersection of the form $V=V_{1} \times\{0\} \subset \mathbb{C}^{p+k}$ for a free divisor $V_{1}$.

Proposition 5.6. Suppose $V=V_{1} \times\{0\} \subset \mathbb{C}^{p+k}$, where $V_{1}, 0 \subset \mathbb{C}^{p}, 0$ is a free divisor and $k>0$. Then, for a versal unfolding of a Morse-type singularity in normal form (1.2), $u \frac{\partial}{\partial u}$ is not $\mathcal{K}_{V}^{*}$-liftable.

Remark 5.7. If $V, 0 \subset \mathbb{C}^{p}, 0$ is smooth, then for any unfolding $F, T \mathcal{K}_{V, u n, e}^{*} \cdot F=$ $T \mathcal{K}_{V, \text { un,e }} \cdot F$. Hence, as in the proof of (4) in Proposition 5.1, any $\mathcal{K}_{V}$-versal unfolding $F$ is $\mathcal{K}_{V}^{*}$-versal, and all $\mathcal{K}_{V}$-liftable vector fields are also $\mathcal{K}_{V}^{*}$-liftable.

Proof of Proposition 5.6. We consider $V=V_{1} \times\{0\} \subset \mathbb{C}^{p+k}$ with $V_{1} \subset \mathbb{C}^{p}$. Let $F(x, u)=(\bar{F}(x, u), u)$ be a versal unfolding of a Morse type singularity in the normal form, where

$$
\bar{F}(x, u)=\left(0, \ldots, 0, x_{1}, \ldots, x_{p^{\prime}}, \sum_{i=p^{\prime}}^{r+p^{\prime}} x_{i}^{2}+u, x_{n-k+1}, \ldots, x_{n}\right) .
$$

Here there are $r$ zeros and $p^{\prime}=p-r$, with $\left(y_{1}, \ldots, y_{p+k}\right)$ denoting coordinates for $\mathbb{C}^{p+k}$. Then, by [D6, Prop. 5.6], $\operatorname{Derlog}(V)$ has generators

$$
\left\{\frac{\partial}{\partial y_{i}}, 1 \leq i \leq r, \quad \zeta_{i}, 0 \leq i \leq p^{\prime}-1\right\}
$$


where $\zeta_{0}$ is the Euler vector field and

$$
\left\{\zeta_{i}\left(y_{p}\right), 1 \leq i \leq p^{\prime}-1\right\} \quad \text { span } m_{p^{\prime}-1} \bmod \left(y_{p^{\prime}}\right)+m_{p}^{2}
$$

Let $M$ denote the submodule of $T \mathcal{K}_{V, \text { un }}^{*} \cdot F$ generated by

$$
\left\{\frac{\partial}{\partial y_{i}}, 1 \leq i \leq r, \quad \frac{\partial}{\partial x_{i}}, n-k+1 \leq i \leq n\right\} .
$$

Then, letting $m_{k}$ denote the ideal of $\mathcal{O}_{\mathbb{C}^{n}, 0}$ generated by $x_{n-k+1}, \ldots, x_{n}$.

$$
T \mathcal{K}_{V, u n, e}^{*} \cdot F /\left(M+m_{k} \theta(\bar{F})\right) \simeq T \mathcal{K}_{h, u n, e} \cdot F_{1} /\left(h \cdot \mathcal{O}_{\mathbb{C}^{n-k+1}, 0}\left\{\frac{\partial}{\partial y_{p}}\right\}\right)
$$

with $h$ the defining equation for $V_{1}$ and $F_{1}(x, u)=\left(\bar{F}_{1}(x, u), u\right)$ where

$$
\bar{F}_{1}(x, u)=\left(x_{1}, \ldots, x_{p^{\prime}-1}, \sum_{i=p^{\prime}}^{n-k+p^{\prime}} x_{i}^{2}+u\right)
$$

We next further divide the RHS of (5.6) by the $\mathcal{O}_{\mathbb{C}^{n-k+1}, 0}$-submodule generated by

$$
\left\{\frac{\partial}{\partial y_{i}}=\frac{\partial \bar{F}_{1}}{\partial x_{i}}, \quad 1 \leq i \leq p^{\prime}-1, \quad \frac{\partial \bar{F}_{1}}{\partial x_{i}}, \quad p^{\prime} \leq i \leq n-k\right\} .
$$

The quotient is isomorphic to

$$
\mathcal{O}_{\mathbb{C}^{p^{\prime}}, 0} / \mathcal{O}_{\mathbb{C}^{p^{\prime}}, 0}\left\{h \circ \bar{F}_{1}, \zeta_{i}\left(y_{p}\right) \circ \bar{F}_{1}, 1 \leq i \leq p^{\prime}-1\right\}
$$

where now $\left(x_{1}, \ldots, x_{p^{\prime}-1}, u\right)$ denote coordinates for $\mathbb{C}^{p^{\prime}}$. As $V$ is not smooth, $h \in$ $m_{p}^{2}$. Lastly, let $Q$ denote the quotient of (5.7) by $m_{p^{\prime}}^{2}$. By the form of $F, Q$ is isomorphic to the quotient of $\mathcal{O}_{\mathbb{C}^{p^{\prime}}} / m_{p^{\prime}}^{2}$ by $\left\{\zeta_{i}\left(y_{p}\right) \circ \bar{F}_{1}\right\}$, which spans a subspace of dimension $p^{\prime}-1$. If $u \frac{\partial \bar{F}}{\partial u}=u \frac{\partial}{\partial y_{p}} \in T \mathcal{K}_{V, u n, e}^{*} \cdot F$, then $u=0$ in $Q$. This would imply by (5.5) that $m_{p^{\prime}}=0$ in $Q$, a contradiction. Thus, $u \frac{\partial}{\partial u}$ is not $\mathcal{K}_{V}^{*}$-liftable.

\section{Morse-Type Singularities for ${ }_{V} \mathcal{K}$-EQuivalence}

We next turn to the situation of equivalences of germs $f_{0}: \mathbb{C}^{n}, 0 \rightarrow \mathbb{C}^{m}, 0$ preserving an analytic germ $V, 0 \subset \mathbb{C}^{n}, 0$ in the source: ${ }_{V} \mathcal{K},{ }_{V} \mathcal{A}$, and ${ }_{V} \mathcal{R}^{+}$equivalences in i) of Table 1 . Before we are ready to prove these results in $\S 7$, we first identify the Morse-type singularities for ${ }_{V} \mathcal{K}$-equivalence. We do so by establishing a duality between Morse-type singularities for ${ }_{V} \mathcal{K}$-equivalence and $\mathcal{K}_{V}$-equivalence.

We compute the extended ${ }_{V} \mathcal{K}$-tangent space for $f_{0}$ by a computation analogous to that for $\mathcal{K}_{V}$-equivalence in [D1]

$$
T_{V} \mathcal{K}_{e} \cdot f_{0}=\mathcal{O}_{\mathbb{C}^{n}, 0}\left\{\zeta_{1}\left(f_{0}\right), \ldots, \zeta_{r}\left(f_{0}\right)\right\}+m_{m} \theta\left(f_{0}\right)
$$

where $\operatorname{Derlog}(V)$ is generated by $\zeta_{1}, \ldots, \zeta_{r}$.

Morse-type singularities for ${ }_{V} \mathcal{K}$-equivalence are germs $g: \mathbb{C}^{n}, 0 \rightarrow \mathbb{C}^{m}, 0$ which have ${ }_{V} \mathcal{K}_{e}$-codim $=1$ and which are ${ }_{V} \mathcal{K}$-equivalent to a germ $f_{0}$ and for a common choice of local coordinates, both $f_{0}$ and $V$ are weighted homogeneous.

In the special case that $V=\mathbb{C}^{n}$, a Morse-type singularity for ${ }_{V} \mathcal{K}$-equivalence is a Morse-type singularity for $\mathcal{K}$-equivalence, and hence is a standard $\Sigma_{n-p+1,0}$ singularity given by the normal form in Example 7.7 of Part II. Next we suppose $V, 0 \subsetneq \mathbb{C}^{n}, 0$. 
Then, as ${ }_{V} \mathcal{K} \subset \mathcal{K}$,

$$
\mathcal{K}_{e}-\operatorname{codim}\left(f_{0}\right) \leq{ }_{V} \mathcal{K}_{e}-\operatorname{codim}\left(f_{0}\right)=1
$$

If $\mathcal{K}_{e}-\operatorname{codim} f_{0}=1$ then, $f_{0}$ is a Morse-type singularity for $\mathcal{K}$-equivalence, and so has corank 1. Also, since ${ }_{V} \mathcal{K}_{e}-\operatorname{codim} f_{0} \geq \operatorname{codim}\left(d f_{0}\left(T_{\text {log }}(V)_{(0)}\right)\right.$, we must have $\operatorname{dim}\left(d f_{0}\left(T_{l o g}(V)_{(0)}\right) \geq m-1\right.$. If it were $m$, then $f_{0}$ would have ${ }_{V} \mathcal{K}_{e}-\operatorname{codim}=0$. Thus, the dimension is $m-1$, and either $\operatorname{corank}\left(f_{0}\right)=1$ or $f_{0}$ is the germ of a submersion. We determine which of the last two conditions occur.

Next, we perform a preliminary reduction modelled on the proof of Lemma 4.12 in Part I (or see Lemma 7.3 in Part II). There is a weighted homogeneous change of coordinates so $V=\mathbb{C}^{r} \times V_{0}$ for $V_{0}, 0 \subset \mathbb{C}^{n^{\prime}}$ with $T_{\text {log }}\left(V_{0}\right)_{(0)}=0$ with both $V_{0}$ and $f_{0}$ weighted homogeneous. If $\operatorname{Derlog}\left(V_{0}\right)$ is generated by $\zeta_{1}, \ldots, \zeta_{s}$, then $\operatorname{Der} \log (V)$ is generated by $\left\{\frac{\partial}{\partial x_{1}}, \ldots, \frac{\partial}{\partial x_{r}}, \zeta_{1}, \ldots, \zeta_{s}\right\}$. Also, $\zeta_{i} \in m_{n} \theta_{n}$ so $\zeta_{i}\left(f_{0}\right) \in m_{n} \theta\left(f_{0}\right)$. Hence, $d f_{0}\left(T_{l o g}(V)_{(0)}\right)$ is spanned by $\left\{\frac{\partial f_{0}}{\partial x_{1}}, \ldots, \frac{\partial f_{0}}{\partial x_{r}}\right\}$. We can find $W \subset \mathbb{C}^{r}$ such that $d f_{0} \mid W$ induces an isomorphism $W \simeq d f_{0}\left(T_{\text {log }}(V)_{(0)}\right)$. There is a complementary subspace $K$ containing $\operatorname{ker}\left(d f_{0}(0)\right)$. In the case corank $\left(f_{0}\right)=1$, then $K=\operatorname{ker}\left(d f_{0}(0)\right)$, while if $f_{0}$ is the germ of a submersion, $\operatorname{ker}\left(d f_{0}(0)\right)$ has codimension 1 in $K$. Define the map $\psi: \mathbb{C}^{n}, 0 \rightarrow \mathbb{C}^{n}, 0$ by $\psi(w, v)=\left(f_{0}(w), v\right)$ for $w \in W$ and $v \in K$. Then, $\psi$ is a germ of a diffeomorphism, preserves $V=\mathbb{C}^{r} \times V_{0}$, and $f_{0} \circ \psi^{-1}\left(x_{1}, \ldots, x_{n}\right)=\left(x_{1}, \ldots, x_{m-1}, g\left(x_{1}, \ldots, x_{n}\right)\right)$. We may further subtract functions in the ideal generated by $x_{1}, \ldots, x_{m-1}$ from $g$, and obtain a weighted homogeneous change of coordinates up to ${ }_{V} \mathcal{K}$-equivalence which leaves $g$ in the form $f_{1}\left(x_{m}, \ldots, x_{n}\right)$.

Thus, after applying ${ }_{V} \mathcal{K}$-equivalence, we obtain for a choice of weights for the coordinates: both $V$ and $f_{0}$ weighted homogeneous, $V=\mathbb{C}^{r} \times V_{0}$ with $V_{0}, 0 \subset \mathbb{C}^{n^{\prime}}$, $T_{l o g}\left(V_{0}\right)_{(0)}=0$, and $f_{0}$ has the preliminary form

$$
f_{0}\left(x_{1}, \ldots, x_{n}\right)=\left(x_{1}, \ldots, x_{m-1}, f_{1}\left(x_{m}, \ldots, x_{n}\right)\right)
$$

If $V \subsetneq \mathbb{C}^{n}$ and $\operatorname{corank}\left(f_{0}\right)=1$, then we claim such germs cannot be Morse-type singularities for ${ }_{V} \mathcal{K}$-equivalence.

Lemma 6.1. If $V, 0 \subsetneq \mathbb{C}^{n}, 0$, then a Morse-type singularity $f_{0}: \mathbb{C}^{n}, 0 \rightarrow \mathbb{C}^{m}, 0$ for ${ }_{V} \mathcal{K}$-equivalence is a germ of a submersion.

Proof. By a change of coordinates, we may assume $f_{0}$ has the normal form (6.3). If $f_{0}$ has corank 1 , then by (6.2), we may assume $f_{0}$ is a Morse-type singularity for $\mathcal{K}$-equivalence so $f_{1} \in m_{n-m+1}^{2}$ with nondegenerate Hessian. If $f_{0}$ has the normal form (6.3), we may suppose $W=\mathbb{C}^{m-1}$. From the form of $T_{V} \mathcal{K}_{e} \cdot f_{0}$ in (6.1), projection onto the last component of $\theta\left(f_{0}\right)$ induces an isomorphism

$$
N_{V} \mathcal{K}_{e} \cdot f_{0} \simeq O_{\mathbb{C}^{n-m+1}, 0} /\left(\frac{\partial f_{1}}{\partial x_{m}}, \ldots, \frac{\partial f_{1}}{\partial x_{r}}, \zeta_{1}\left(f_{1}\right), \ldots, \zeta_{s}\left(f_{1}\right)\right)
$$

where $\mathbb{C}^{n-m+1}$ denotes $\{0\} \times \mathbb{C}^{n-m+1}$. However, $\zeta_{i} \in m_{n} \theta_{n}$ implies $\zeta_{i}\left(f_{1}\right) \in m_{n}^{2}$. Also $\frac{\partial f_{1}}{\partial x_{i}} \in m_{n}$, so by $(6.4)$

$$
\operatorname{dim} N_{V} \mathcal{K}_{e} \cdot f_{0} \geq 1+\operatorname{dim}_{\mathbb{C}}\left(m_{n-m+1} /\left(\left\langle\frac{\partial f_{1}}{\partial x_{m}}, \ldots, \frac{\partial f_{1}}{\partial x_{r}}\right\rangle+m_{n}^{2}\right)\right)
$$

Hence, if $r<n$, then ${ }_{V} \mathcal{K}_{e}-\operatorname{codim} f_{0} \geq 2$. Thus, $r=n$ and $V=\mathbb{C}^{n}$. 
Thus, for ${ }_{V} \mathcal{K}$-equivalence with $V \subsetneq \mathbb{C}^{n}$, the Morse-type singularities are germs of submersions. We now establish a dual correspondence between $\mathcal{K}_{V}$ and ${ }_{V} \mathcal{K}-$ equivalence for such germs.

Lemma 6.2 (Dual Correspondence for $\mathcal{K}_{V}$ and $\left.{ }_{V} \mathcal{K}\right)$. Let $f_{0}: \mathbb{C}^{n}, 0 \rightarrow \mathbb{C}^{m}, 0$ be a germ of a submersion, so $X_{0}=f_{0}^{-1}(0)$ is smooth. Let $g_{0}: \mathbb{C}^{n-m}, 0 \rightarrow \mathbb{C}^{n}, 0$ be a germ of an immersion which parametrizes $X_{0}$. If $V, 0 \subset \mathbb{C}^{n}, 0$, then there is a natural isomorphism of $\mathcal{O}_{X_{0}, 0}-$ modules

$$
N_{V} \mathcal{K}_{e} \cdot f_{0} \simeq N \mathcal{K}_{V, e} \cdot g_{0}
$$

Proof. The isomorphism is induced by extending $\xi \in \theta\left(g_{0}\right)$ to a $\tilde{\xi} \in \theta_{n}$ and sending it to $\tilde{\xi}\left(f_{0}\right)_{\mid X_{0}}$ To see that this is well defined and induces an isomorphism, we change coordinates so we may assume that $f_{0}$ is the projection $\pi: \mathbb{C}^{n} \rightarrow \mathbb{C}^{m}=\{0\} \times \mathbb{C}^{m}$ and $g_{0}$ is the immersion $i: \mathbb{C}^{n-m}=\mathbb{C}^{n-m} \times\{0\} \hookrightarrow \mathbb{C}^{m}$. Let $\operatorname{Derlog}\left(V_{0}\right)$ be generated by $\zeta_{1}, \ldots, \zeta_{s}$, and express $\zeta_{i}=\zeta_{i 1}+\zeta_{i 2}$ relative to the decomposition $\mathbb{C}^{n}=\mathbb{C}^{n-m} \oplus \mathbb{C}^{m}$. Then, $\zeta_{i}(\pi)=\zeta_{i 2}(\pi)=\zeta_{i 2}$, so

$$
N_{V} \mathcal{K}_{e} \cdot \pi \simeq O_{\mathbb{C}^{n-m}, 0}\left\{\frac{\partial}{\partial x_{n-m+1}}, \ldots, \frac{\partial}{\partial x_{n}}\right\} / O_{\mathbb{C}^{n-m}, 0}\left\{\zeta_{i 2}\right\}
$$

Also, $\frac{\partial i}{\partial x_{j}}=\frac{\partial}{\partial x_{j}}$ for $1 \leq j \leq n-m$, and $\pi \circ \zeta_{j} \circ i=\zeta_{j 2}$. Hence, we likewise obtain

$$
N \mathcal{K}_{V, e} \cdot i \simeq O_{\mathbb{C}^{n-m}, 0}\left\{\frac{\partial}{\partial x_{n-m+1}}, \ldots, \frac{\partial}{\partial x_{n}}\right\} / O_{\mathbb{C}^{n-m}, 0}\left\{\zeta_{i 2}\right\}
$$

We note (6.7) and (6.8) are isomorphic via the map $\xi \in \theta(i) \mapsto \tilde{\xi}(\pi)_{\mid \mathbb{C}^{n-m}}$, which is independent of the extension of $\xi$ to $\tilde{\xi}$ on $\mathbb{C}^{n}$.

Remark . Even in the case $n=m$, this is still formally correct provided we allow maps $\mathbb{C}^{0}=\{0\} \rightarrow \mathbb{C}^{n}, 0$.

Example 6.3. If $\mathbb{C}^{3}$ has coordinates $(x, y, z)$ and $V, 0 \subset \mathbb{C}^{3}, 0$ is the $x y$-plane, then the inclusion of the surface $z=x^{2}-y^{2}$ is a Morse-type singularity for $\mathcal{K}_{V^{-}}$ equivalence, and the function $f_{0}(x, y, z)=z-\left(x^{2}-y^{2}\right)$ is a Morse-type singularity for ${ }_{V} \mathcal{K}$-equivalence as in figure 1.

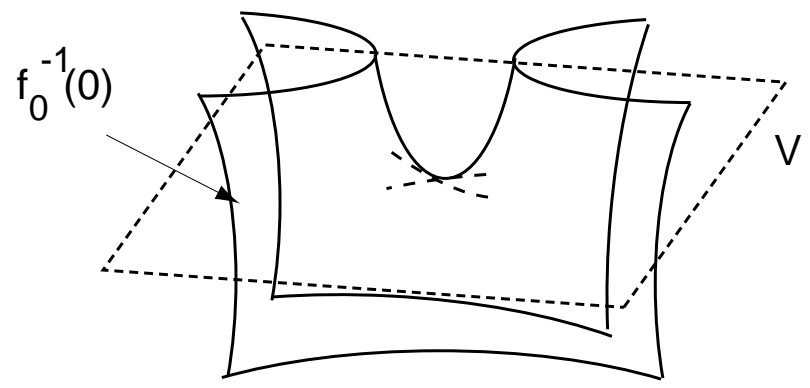

Figure 1. Morse Type Singularity $f_{0}$ for ${ }_{V} \mathcal{K}$-equivalence for $V$ smooth

Lemma 6.2 allows us to move between Morse-type singularities for $\mathcal{K}_{V}$ and ${ }_{V} \mathcal{K}$ equivalence. If $V, 0 \subset \mathbb{C}^{n}, 0$ has a Morse-type singularity $f_{0}: \mathbb{C}^{n}, 0 \rightarrow \mathbb{C}^{m}, 0$ for 
${ }_{V} \mathcal{K}$-equivalence, then by Lemma $6.2, V, 0$ has Morse-type singularities for $\mathcal{K}_{V^{-}}$ equivalence in dimension $n-m$, and hence in all allowable dimensions by Corollary 7.4 of Part II. Conversely, suppose $V, 0$ has Morse-type singularities for $\mathcal{K}_{V^{-}}$ equivalence in all allowable dimensions. Given $m<n$, there is for appropriate local coordinates a Morse type singularity for $\mathcal{K}_{V}$-equivalence given as in the normal form (1.1).

$$
\begin{aligned}
g_{0}\left(x_{1}, \ldots, x_{n-m}\right)=\left(0, \ldots, 0, x_{1}, \ldots, x_{n^{\prime}-1}, Q\left(x_{n^{\prime}}, \ldots, x_{n-m}\right)\right) & \\
\text { where } \quad Q\left(x_{n^{\prime}}, \ldots, x_{n-m}\right) & =\sum_{j=n^{\prime}}^{n-m} x_{j}^{2}
\end{aligned}
$$

We can modify the normal form so it is still a Morse type singularity except it is a now the germ of an immersion

$$
g_{1}\left(x_{1}, \ldots, x_{n-m}\right)=\left(0, \ldots, 0, x_{n^{\prime}}, \ldots, x_{n-m}, x_{1}, \ldots, x_{n^{\prime}-1}, Q\left(x_{n^{\prime}}, \ldots, x_{n-m}\right)\right)
$$

Note that we will have $m-1$ " 0 's". Then, we obtain a Morse-type singularity for ${ }_{V} \mathcal{K}$-equivalence for the same coordinates as

$$
f_{0}\left(y_{1}, \ldots, y_{n}\right)=\left(y_{1}, \ldots, y_{m-1}, y_{n}-\sum_{j=m}^{r} y_{j}^{2}\right)
$$

Next, we observe that (6.10) is the general normal form for Morse-type singularities for ${ }_{V} \mathcal{K}$-equivalence.

Lemma 6.4 (Local Normal Form). Let $f_{0}: \mathbb{C}^{n}, 0 \rightarrow \mathbb{C}^{m}, 0$ with $n \geq m$ be a Morse-type singularity for $V, 0 \subsetneq \mathbb{C}^{n}, 0$. Then, up to ${ }_{V} \mathcal{K}$-equivalence, we may assume $V, 0=\mathbb{C}^{r} \times V_{0}, 0$ for $V_{0}, 0 \subset \mathbb{C}^{n^{\prime}}, 0$ with $T_{\text {log }}\left(V_{0}\right)_{(0)}=0$, and with respect to coordinates for which $V_{0}, 0$ and $f_{0}$ are weighted homogeneous, $f_{0}$ is given by

$$
\left.f_{0}\left(x_{1}, \ldots, x_{n}\right)=\left(x_{1}, \ldots, x_{m-1}, x_{n}-\sum_{j=m}^{r} x_{j}^{2}\right)\right)
$$

(here $x_{n}$ is a coordinate for $\mathbb{C}^{n^{\prime}}$ so that $\left\{x_{n}, \zeta_{1}\left(x_{n}\right), \ldots, \zeta_{s}\left(x_{n}\right)\right\}$ generate $m_{n^{\prime}}$ ).

Proof. We have already established the preliminary normal form in (6.3). We may choose coordinates for $\mathbb{C}^{n^{\prime}}$ so that $\frac{\partial f_{1}}{\partial x_{n}}(0) \neq 0$ but $\frac{\partial f_{1}}{\partial x_{j}}(0)=0$ for $j=m, \ldots, n-1$. Hence, absorbing the coefficient of $x_{n}, f_{1}=x_{n}+h$ with $h \in m_{n-m+1}^{2}$. Thus, $T_{V} \mathcal{K}_{e} \cdot f_{0}$ contains

$$
\mathcal{O}_{\mathbb{C}^{n}, 0}\left\{\frac{\partial}{\partial y_{1}}, \ldots, \frac{\partial}{\partial y_{m-1}}\right\}+m_{m-1} \mathcal{O}_{\mathbb{C}^{n}, 0}\left\{\frac{\partial}{\partial y_{m}}\right\}
$$

Thus, projecting along this subspace onto $\mathcal{O}_{\mathbb{C}^{n-m+1}, 0}\left\{\frac{\partial}{\partial y_{m}}\right\} \simeq \mathcal{O}_{\mathbb{C}^{n-m+1}, 0}$, we obtain the image of $T_{V} \mathcal{K}_{e} \cdot f_{0}$ to be

$$
\left\langle x_{n}, \frac{\partial h}{\partial x_{m}}, \ldots, \frac{\partial h}{\partial x_{r}}, \zeta_{1}\left(x_{n}\right), \ldots, \zeta_{s}\left(x_{n}\right)\right\rangle \bmod \left(m_{n-m+1}^{2}\right)
$$

where $x_{n}$ is obtained from $f_{1}$.

Hence, $f_{0}$ will have ${ }_{V} \mathcal{K}_{e}$-codimension 1 exactly when

$$
x_{n}, \frac{\partial h}{\partial x_{m}}, \ldots, \frac{\partial h}{\partial x_{r}}, \zeta_{1}\left(x_{n}\right), \ldots, \zeta_{s}\left(x_{n}\right) \operatorname{span} m_{n-m+1} \bmod \left(m_{n-m+1}^{2}\right)
$$


Since $x_{n}, \zeta_{1}\left(x_{n}\right), \ldots, \zeta_{s}\left(x_{n}\right) \in m_{n^{\prime}}$, and $n-m+1=n^{\prime}+(r-(m-1)),(6.12)$ will hold exactly when $x_{n}, \zeta_{1}\left(x_{n}\right), \ldots, \zeta_{s}\left(x_{n}\right)$ spans $m_{n^{\prime}}$ and $\frac{\partial h}{\partial x_{m}}, \ldots, \frac{\partial h}{\partial x_{r}}$ spans $m_{n-m+1} /\left(m_{n^{\prime}}+m_{n-m+1}^{2}\right)$.

Then, by Nakayama's Lemma,

$$
\begin{aligned}
& T_{V} \mathcal{K} \cdot f_{0}=\left(x_{1}, \ldots, x_{m-1}, x_{r+1}, \ldots, x_{n}\right) \mathcal{O}_{\mathbb{C}^{n}, 0}\left\{\frac{\partial}{\partial y_{m}}\right\} \\
&+m_{n} \mathcal{O}_{\mathbb{C}^{n}, 0}\left\{\frac{\partial}{\partial y_{1}}, \ldots, \frac{\partial}{\partial y_{m-1}}\right\}+m_{n}^{2} \mathcal{O}_{\mathbb{C}^{n}, 0}\left\{\frac{\partial}{\partial y_{m}}\right\}
\end{aligned}
$$

All such germs $f_{0}^{\prime}$ in the preliminary form (6.3) with the same 1-jet have the same tangent space. Thus, we may apply Mather's geometric Lemma to conclude that $f_{0}$ is ${ }_{V} \mathcal{K}$-equivalent to its linearization, establishing the lemma.

Remark . If $n=m$, then the Morse-type singularity for $V=\mathbb{C}^{n-1}$ (so $r=n-1$ ) is $f_{0}=i d$, which has ${ }_{V} \mathcal{K}_{e}-\operatorname{codim}=1$. The nontrivial deformation of $f_{0}$ moves the image of $V$ off 0 .

Having established the normal form, the arguments given in $\S 4$ of Part I allow us to deduce the analogues of Corollary 7.4 of Part II.

\section{Corollary 6.5.}

(1) If $V, 0 \subset \mathbb{C}^{p}, 0$ has a Morse type singularity $f_{0}: \mathbb{C}^{n}, 0 \rightarrow \mathbb{C}^{m}, 0$ for ${ }_{V} \mathcal{K}$ equivalence, then it has Morse-type singularities for ${ }_{V} \mathcal{K}$-equivalence for all "allowable dimensions" $m \leq r+1$ where $r=\operatorname{dim}\left(T_{l o g}(V)_{0}\right)$.

(2) Suppose $V, 0=\mathbb{C}^{r} \times V_{0}, 0$ is weighted homogeneous with $V_{0}, 0 \subset \mathbb{C}^{n^{\prime}}, 0$ and $\left(T_{\text {log }}\left(V_{0}\right)_{(0)}\right)=(0)$. Then $V$ has Morse type singularities in all allowable dimensions iff there is a weighted hyperplane in $\mathbb{C}^{p^{\prime}}$ which is transverse to the orbits of $A u t_{1}\left(V_{0}\right)$ in a punctured neighborhood of 0 . Here Aut $t_{1}\left(V_{0}\right)$ denotes the group of linearized automorphisms of $V_{0}$.

(3) If $V, 0 \subset \mathbb{C}^{n}, 0$ has Morse type singularities for ${ }_{V} \mathcal{K}$-equivalence, then there is a Zariski open dense subset of $\Sigma_{n-m}$ consisting of jets of Morse-type singularities (here $\Sigma_{n-m}$ denotes the 2-jets of germs of submersions $f_{0}$ for which $\operatorname{ker}\left(d f_{0}(0)\right)$ is not algebraically transverse to $V$ at 0$)$.

Also, because of the correspondence between Morse-type singularities for $\mathcal{K}_{V}$ and ${ }_{V} \mathcal{K}$-equivalence, we also obtain the analogues of Proposition 7.5 of Part II for products and the version of Lemma 4.29 of Part I for product unions. Also,we have an analogue of Lemma 7.8 of Part II. We define for $f_{0}$ in normal form (6.11) the exceptional weight type to be $\operatorname{wt}\left(y_{n}\right)$ which is the same as for $g_{0}$. The versal unfolding $F$ of $f_{0}$ given by

$$
F(x, u)=(\bar{F}(x, u), u) \quad \text { with } \quad \bar{F}(x, u)=f_{0}(x)+(0, \ldots, 0, u)
$$

Lemma 6.6. For the Morse-type singularity in the normal form (6.11), with nonzero exceptional weight type, with versal unfolding $F$ given by (6.14), the vector field $u \frac{\partial}{\partial u}$ is ${ }_{V} \mathcal{K}$-liftable. 


\section{Equivalence of Mappings while Preserving a Free Divisor}

We now apply our analysis of Morse-type singularities for ${ }_{V} \mathcal{K}$-equivalence to prove Theorems 1 - 4 .

Let $V, 0 \subset \mathbb{C}^{n}, 0$ a free divisor. In order to prove Theorem 1 we will still use the strategy (1.3) even though we consider ${ }_{V} \mathcal{K}$ instead of $\mathcal{K}_{V}$. To apply it, we will establish the following Propositions.

Proposition 7.1. Suppose that $V, 0 \subset \mathbb{C}^{n}$ is a free divisor. If $0 \leq n-m<h n(V)$, then the group ${ }_{V} \mathcal{K}$ acting on germs $f_{0}: \mathbb{C}^{n}, 0 \rightarrow \mathbb{C}^{m}, 0$ is Cohen-Macaulay.

Proposition 7.2. Under the same assumptions as Proposition 7.1, suppose that moreover $V$ generically has Morse-type singularities for $\mathcal{K}_{V}$-equivalence in dimension $n-m$. Then, $a_{V} \mathcal{K}$-versal unfolding $F$ of a germ $f_{0}: \mathbb{C}^{n}, 0 \rightarrow \mathbb{C}^{m}, 0$ generically has ${ }_{V} \mathcal{K}$-liftable vector fields.

Using these propositions, we can apply Theorem 1 of Part II to immediately deduce Theorem 1.

Proof of Proposition 7.1. We first check that ${ }_{V} \mathcal{K}$ has geometrically defined discriminants. The group ${ }_{V} \mathcal{K}$ induces a local action at points $x$ near 0 which is again of the same form ${ }_{(V, x)} \mathcal{K}$. Also, the ${ }_{V} \mathcal{K}$-discriminant for an unfolding $F$ consists of parameter values $u \in \mathbb{C}^{q}$ such that either $X_{u}=F_{u}^{-1}(0)$ is not smooth or it is, but it fails to be transverse to $V$. We note ${ }_{V} \mathcal{K}$-liftable diffeomorphisms lift to ${ }_{V} \mathcal{K}_{e q}$-equivalences which preserve these properties (where ${ }_{V} \mathcal{K}_{e q}$-equivalences are ${ }_{V} \mathcal{K}$-equivalences of unfoldings which also allow change of coordinates in the parameters in $\mathbb{C}^{q}$ ). Thus, ${ }_{V} \mathcal{K}$ has geometrically defined discriminants.

Second, we apply an argument using Eagon-Northcott [EN] analogous to that in Proposition 4.3. The normal space $N_{V} \mathcal{K}_{u n, e} \cdot F$, viewed as an $\mathcal{O}_{\mathcal{X}, 0}$-module, where $\mathcal{X}=\bar{F}^{-1}(0)$ has pushforward which is Cohen-Macaulay and whose support is the discriminant $\left.D_{V} \mathcal{K}(F)\right)=\pi\left(C_{V} \mathcal{K}(F)\right)$ of codimension 1. Thus, ${ }_{V} \mathcal{K}$ is CohenMacaulay.

Proof of Proposition 7.2. We will apply the criterion (1.3), even though we work with ${ }_{V} \mathcal{K}$ instead of a subgroup of $\mathcal{K}_{V}$. Also, by proposition $7.1,{ }_{V} \mathcal{K}$ itself is CohenMacaulay, so we apply the criterion to ${ }_{V} \mathcal{K}$ rather than a CM-reduction.

By the above remarks (1) of (1.3) is satisfied. Next, by Lemma 6.2, the genericity of Morse type singularities for $V$ in dimension $n-m$ implies that all points $x$ on the canonical Whitney strata of $V$ of codimension $\leq n-m+1$ there are Morse-type singularities of nonzero exceptional weight type $f_{0}: \mathbb{C}^{n}, x \rightarrow \mathbb{C}^{m}, 0$ for $(V, x) \mathcal{K}$-equivalence. Second, just as for $\mathcal{K}_{V}$-equivalence, we can still perturb a finite codimension germ $f_{0}$ so it only has one ${ }_{V} \mathcal{K}$-critical point on $V$ and at that point it is a Morse-type singularity. This establishes (2) of (1.3) and allows us to consider the criterion for ${ }_{V} \mathcal{K}$-equivalence.

For (3) of (1.3) we observe that $f_{0}: \mathbb{C}^{n}, 0 \rightarrow \mathbb{C}^{m}, 0$ has ${ }_{V} \mathcal{K}_{e}$-codimension 0 iff $f_{0}$ is the germ of a submersion (i.e. $\mathcal{K}_{e}$-codimension 0 ) and $X_{0}=f_{0}^{-1}(0)$ is algebraically transverse to $V$ at 0 .

Lastly, any stratum of codimension $>n-m+1$ lies in the closure of a stratum of codimension $=n-m+1$. Hence, by Corollary 6.5 , we have the Zariski open subset $\mathcal{W}$ of $\Sigma_{n-m}$. Also, by Lemma 6.6, for the normal form (6.11), $u \frac{\partial}{\partial u}$ is liftable. Thus, (4) of (1.3) follows. 
Hence, by Proposition 1.1 applied to ${ }_{V} \mathcal{K}$ (whose proof applies using the above observation) we deduce the genericity of ${ }_{V} \mathcal{K}$-liftable vector fields.

Then, Theorem 2 follows from Theorem 1, using the following Lemma relating ${ }_{V} \mathcal{A}$-stable germs and ${ }_{V} \mathcal{K}$-versal unfoldings.

Lemma 7.3. Suppose that $V, 0 \subset \mathbb{C}^{n}$ is a free divisor, and that $f_{0}: \mathbb{C}^{n}, 0 \rightarrow \mathbb{C}^{m}, 0$ is ${ }_{V} \mathcal{A}$-stable. Then, the unfolding $F(x, u)=(f(x)-u, u): \mathbb{C}^{n+m}, 0 \rightarrow \mathbb{C}^{2 m}, 0$ is ${ }_{V} \mathcal{K}$-versal and the ${ }_{V} \mathcal{A}$-discriminant of $f_{0}$ equals the ${ }_{V} \mathcal{K}$-discriminant of $F$.

Proof. The proof is very similar to that of Mather [M-IV] or Martinet [Mar] for ordinary $\mathcal{A}$ and $\mathcal{K}$ equivalence. By the versality theorem, ${ }_{V} \mathcal{A}$-stability under deformations, which is equivalent to $f_{0}$ being its own versal deformation, is equivalent to infinitesimal ${ }_{V} \mathcal{A}$-versality.

$$
\mathcal{O}_{\mathbb{C}^{n}, 0}\left\{\zeta_{1}\left(f_{0}\right), \ldots, \zeta_{n}\left(f_{0}\right)\right\}+\mathcal{O}_{\mathbb{C}^{m}, 0}\left\{\frac{\partial}{\partial y_{1}}, \ldots, \frac{\partial}{\partial y_{m}}\right\}=\theta\left(f_{0}\right)
$$

where $\left\{\zeta_{1}, \ldots, \zeta_{n}\right\}$ generate $\operatorname{Derlog}(V)$. By the preparation theorem, this is equivalent to

$$
\mathcal{O}_{\mathbb{C}^{n}, 0}\left\{\zeta_{1}\left(f_{0}\right), \ldots, \zeta_{n}\left(f_{0}\right)\right\}+m_{m} \theta\left(f_{0}\right)+\left\langle\frac{\partial}{\partial y_{1}}, \ldots, \frac{\partial}{\partial y_{m}}\right\rangle=\theta\left(f_{0}\right)
$$

which (since $\frac{\partial \bar{F}}{\partial u_{i}}=\frac{\partial}{\partial y_{i}}$ ) is the infinitesimal condition that $F$ is ${ }_{V} \mathcal{K}$-versal.

Finally, we verify that the discriminants agree. First, $\mathcal{X}=\bar{F}^{-1}(0)$ is smooth and $\pi: \mathcal{X} \rightarrow \mathbb{C}^{m}$ is $\mathcal{A}$-equivalent to $f_{0}$ via a diffeomorphism $\varphi(x)=\left(x, f_{0}(x)\right)$ sending $V$ to $V \cap \mathcal{X}$. Then, $u \in D_{V} \mathcal{K}(F)$ if either $X_{u}=F_{u}^{-1}(0)=\mathcal{X} \cap\left(\mathbb{C}^{n} \times\{u\}\right)$ is not smooth or it is, but is not transverse to $V$. Equivalently, $u$ is a critical point of $\pi \mid \mathcal{X}$ or it is a regular point but $\pi^{-1}(u) \cap \mathcal{X}$ is not transverse to $V \times \mathbb{C}^{m}$. Under the equivalence with $f_{0}$, this is the condition that $y(=u) \in D_{V \mathcal{A}}(F)$, and conversely.

Furthermore, Corollary 3 is an immediate consequence of Theorem 2.

Proof of Theorem 4. Lastly, we turn to the ${ }_{V} \mathcal{A}$-bifurcation set for germs $f_{0}$ : $\mathbb{C}^{n}, 0 \rightarrow \mathbb{C}^{p}, 0$. Let $F(x, u)=(\bar{F}(x, u), u): \mathbb{C}^{n+q}, 0 \rightarrow \mathbb{C}^{p+q}, 0$ denote the ${ }_{V} \mathcal{A}^{-}$ versal unfolding of $f_{0}$. Then, the $V \times \mathbb{C}^{q} \mathcal{A}$-discriminant $D_{V \times \mathbb{C} q} \mathcal{A}(F)$ of $F$, viewed as a germ, is a free divisor by Theorem 2 .

We can repeat the reasoning in [D7] to show that the results of that paper apply equally well to ${ }_{V} \mathcal{A}$-equivalence and its relation with $\mathcal{K}_{D^{-}}$equivalence where $D=D_{V \mathcal{A}}(F)$. For a germ $f_{0}: \mathbb{C}^{n}, 0 \rightarrow \mathbb{C}^{p}, 0$ and an analytic germ $V, 0 \subset \mathbb{C}^{n}, 0$, let the $V \mathcal{A}$-versal unfolding again be $F: \mathbb{C}^{n+q}, 0 \rightarrow \mathbb{C}^{p+q}, 0$. In particular, if $g_{0}: \mathbb{C}^{p}, 0 \rightarrow \mathbb{C}^{p+q}, 0$ denotes the inclusion, then any unfolding $f$ of $f_{0}$ is obtained up to ${ }_{V} \mathcal{A}$-equivalence as the pullback of $F$ by an unfolding $g$ of $g_{0}$. Moreover, we conclude the following.

Theorem 7.4. In the preceding situation (without requiring that $V$ be a free divisor),

(1) $f_{0}$ has finite ${ }_{V} \mathcal{A}$-codimension iff $g_{0}$ has finite $\mathcal{K}_{D}$-codimension;

(2) if either is finite, then there is the isomorphism of $\mathcal{O}_{\mathbb{C}^{p}, 0}$-modules

$$
N_{V} \mathcal{A}_{e} \cdot f_{0} \simeq N \mathcal{K}_{D, e} \cdot g_{0}
$$

Let $g$ be an unfolding of $g_{0}$, with pullback of $F$ denoted by $f$. 


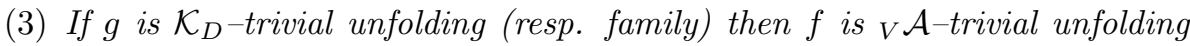
(resp. family);

(4) $g$ is $\mathcal{K}_{D}$-versal iff $f$ is ${ }_{V} \mathcal{A}$-versal.

Then, it follows that the ${ }_{V} \mathcal{A}$-bifurcation set of $F$ is the $\mathcal{K}_{D^{-}}$-discriminant $D_{D}(G)$, where $G$ is the $\mathcal{K}_{D^{-}}$-versal unfolding of $g_{0}$. Now, in our case, $F$ is $V \times \mathbb{C} q \mathcal{A}$-stable. Moreover, $0<n-p<h n\left(V \times \mathbb{C}^{q}\right)=h n(V)$ (in addition $V \times \mathbb{C}^{q}$ has Morse type singularities in dimension $n-p$, which is equivalent to $V$ having Morse type singularities in dimension $n-p)$. Hence, by Theorem 2, the $V \times \mathbb{C}^{q} \mathcal{A}$-discriminant of $F$ in $\mathbb{C}^{p+q}$ is a free divisor.

The assumption that $f_{0}$ belongs to the ${ }_{V} \mathcal{A}$-distinguished bifurcation class, together with Theorem 7.4 implies: i) the strata of $D_{D}(G)$ correspond to the ${ }_{(V, x)} \mathcal{K}-$ classes; ii) the strata of codimension $\geq p+1$ belong in the closure of strata of codimension $p+1$; strata of codimension $\leq p+1$ generically have Morse type singularities; and the strata of $D_{D}(G)$ of codimension $\leq p$ are made up of ${ }_{(V, x)} \mathcal{K}-$ multigerm classes, and are hence holonomic. Thus, we can apply Theorem 1 of Part I to conclude that $D_{D}(G)=B_{V \mathcal{A}}(F)$ is a free divisor defined by the module of $\mathcal{K}_{D}$ (i.e. $\left.V \times \mathbb{C}^{q} \mathcal{A}\right)$-liftable vector fields.

\section{Germs on a Fixed Free Complete Intersection}

The proofs of Theorems 7, 8 and Corollary 9 follow arguments similar to those given already, and make use of the results of $\S 4$ and 5 . This is why we have postponed the proofs until now.

We begin by defining a CM-reduction for $\mathcal{K}(V)$. We first assume $V, 0 \subset \mathbb{C}^{n}, 0$ is a free complete intersection of dimension $k$ with free defining equation $h: \mathbb{C}^{n}, 0 \rightarrow$ $\mathbb{C}^{n-k}, 0$, so that $\operatorname{Derlog}(h)$ is freely generated by $\left\{\zeta_{1}, \ldots, \zeta_{k}\right\}$. We let $\mathcal{K}(V)^{*}$ denote the subgroup of $\mathcal{K}(V)$ for which the diffeomorphism of $\mathbb{C}^{n}, 0$ restricted to $V$ is a restriction of a diffeomorphism preserving the level sets of $h$. This again gives a geometric subgroup using the arguments in [D4, §9-11]. Then, as in [D4, §9-11], we compute for $f_{0}: \mathbb{C}^{n}, 0 \rightarrow \mathbb{C}^{p}, 0$,

$$
T \mathcal{K}(V)^{*} \cdot f_{0}=\mathcal{O}_{\mathbb{C}^{n}, 0}\left\{\zeta_{1}\left(f_{0}\right), \ldots, \zeta_{k}\left(f_{0}\right)\right\}+\left(h^{*} m_{n-k}+f_{0}^{*} m_{p}\right) \theta\left(f_{0}\right)
$$

If $F: \mathbb{C}^{n+q}, 0 \rightarrow \mathbb{C}^{p+q}, 0$ is a $\mathcal{K}(V)^{*}$-versal unfolding of $f_{0}$, it is also a $\mathcal{K}(V)$-versal unfolding. Then, the unfolding tangent space is given by

$$
T \mathcal{K}(V)_{u n, e}^{*} \cdot F=\mathcal{O}_{\mathbb{C}^{n}, 0}\left\{\zeta_{1}(\bar{F}), \ldots, \zeta_{k}(\bar{F})\right\}+\left(h^{*} m_{n-k}+\bar{F}^{*} m_{p}\right) \theta(\bar{F})
$$

We let $X=\bar{F}^{-1}(0)$ which is a complete intersection algebraically transverse to $V \times \mathbb{C}^{q}$. Hence, $\mathcal{X}=X \cap\left(V \times \mathbb{C}^{q}\right)$ is still a complete intersection of dimension $k+q-p$. Then, by (8.2) the extended normal space for the unfolding is given by

$$
N \mathcal{K}(V)_{u n, e}^{*} \cdot F \simeq \mathcal{O}_{\mathcal{X}, 0}^{(p)} / \mathcal{O}_{\mathcal{X}, 0}\left\{\zeta_{1}(\bar{F}), \ldots, \zeta_{k}(\bar{F})\right\}
$$

The critical sets $C_{\mathcal{K}(V)}(F)$ for $\mathcal{K}(V)$, and $C_{\mathcal{K}(V)^{*}}(F)$ for $\mathcal{K}(V)^{*}$ are given by

$$
C_{\mathcal{K}(V)}(F)=\operatorname{supp}\left(N \mathcal{K}(V)_{\text {un,e }} \cdot F\right) \quad \text { and } \quad C_{\mathcal{K}(V)^{*}}(F)=\operatorname{supp}\left(N \mathcal{K}(V)_{\text {un,e }}^{*} \cdot F\right)
$$

Then, there is the analogue of Proposition 4.1.

Proposition 8.1. For a free complete intersection $V$ : If $n-p<h(V)$

(1) i) $f_{0}: \mathbb{C}^{n}, 0 \rightarrow \mathbb{C}^{p}, 0$ has finite $\mathcal{K}(V)$-codimension iff it has finite $\mathcal{K}(V)^{*}$ codimension. 
(2) ii) For an unfolding $F$ of $f_{0}$,

$$
C_{\mathcal{K}(V)}(F)=C_{\mathcal{K}(V)^{*}}(F)
$$

The proof that is analogous to that of Proposition 4.1.

That the $\mathcal{K}(V)$-discriminant is geometrically defined is straightforward, and applying Eagon-Northcott as in Proposition 4.3 so we conclude that $\mathcal{K}(V)^{*}$ is a CMreduction of $\mathcal{K}(V)$. We may apply Theorem 2 of Part II to conclude $D_{\mathcal{K}(V)}(F)$ is a free* divisor defined by the module of $\mathcal{K}(V)^{*}$-liftable vector fields.

Having proven Theorem 7 , we can obtain Theorem 8 exactly as we obtained Theorem 2 from Theorem 1 by applying an analogue of Lemma 7.3.

Lemma 8.2. Suppose that $V, 0 \subset \mathbb{C}^{n}$ is a free divisor, and that $f_{0}: \mathbb{C}^{n}, 0 \rightarrow$ $\mathbb{C}^{p}, 0$ is $\mathcal{A}(V)$-stable. Then, the unfolding $F(x, u)=(f(x)-u, u): \mathbb{C}^{n+p}, 0 \rightarrow$ $\mathbb{C}^{2 p}, 0$ is $\mathcal{K}(V)$-versal and the $\mathcal{A}(V)$-discriminant of the germ $f_{0}$ equals the $\mathcal{K}(V)$ discriminant of the unfolding $F$.

Finally Corollary 9 follows as explained in $\S 3$.

\section{Discussion of the Consequences for Understanding Discriminants}

The results obtained in this paper, including those from the earlier parts I and II which generalize many earlier results, give a broad view of how generally discriminants and bifurcation sets for various equivalences are free divisors. Several questions raised by the referee include the following. Do the free divisors which arise for the additional equivalences really give new free divisors? Can we determine based on the properties of a free divisor whether it arises as a discriminant for a specific equivalence? Do sporadic free divisors which have been identified by adhoc methods arise as discriminants?

In fact, we are a long way from answering in any effective way these questions. However, we can draw a few conclusions.

For $\mathcal{K}_{V^{-}}$equivalence with $V$ the simplest free divisor $\{0\} \subset \mathbb{C}$, resp. free complete intersection $\{0\} \subset \mathbb{C}^{p}$, the $\mathcal{K}_{V}$-discriminants are discriminants for isolated hypersurface singularities, resp. isolated complete intersection singularities, which are free divisors. Next, taking $V$ to be these divisors, the $\mathcal{K}_{V}$-discriminants are the bifurcation sets for $\mathcal{A}$-versal unfoldings, and are free divisors provided we are in the distinguished bifurcation class. This progression from $\{0\}$ to discriminants of stable unfoldings to bifurcation sets represents increasingly complicated structure (at least by measures such as topological interpretation of codimension [DM] and higher multiplicities [D2]). Equivalences which introduce the behavior relative to these free divisors should also further increase the complexity.

While certain equivalences such as ${ }_{V} \mathcal{K}$-equivalence of germs which are submersions will have the same discriminants as the $\mathcal{K}_{V}$-equivalence of a corresponding immersion germ by the correspondence of $\S 6$, Arnold has shown that simple boundary singularities give bifurcation sets associated to Weyl groups and Coxeter groups other than the A, D, E groups associated to the simple hypersurface singularities, indicating new complexity.

Although there is considerable knowledge about the local structure of discriminants for stable germs in low codimension, there is as yet no systematic method to characterize the discriminants belonging to a given equivalence, nor to characterize the complexity. Now that we have seen the abundance of free divisors, these questions are important ones to consider. 


\section{REFERENCES}

[A1] Arnol'd, V. I. Wave front evolution and equivariant Morse lemma Comm. Pure App. Math. 29 (1976), 557-582

[A2] Indices of singular 1-forms on a manifold with boundary, convolution of invariants of reflection groups, and singular projections of smooth surfaces Russian Math. Surveys 34 (1979), 1-42

[Br] Bruce, J.W. Vector fields on discriminants and bifurcation varieties London Math. Soc. 17 (1985), 257-262

[BG1] Bruce, J.W. and Giblin, P.J. Projections of Surfaces with Boundaries Proc. London Math. Soc. 60 (1990), 392-416

[BG2] - Smooth maps of discriminant varieties Proc. London Math. Soc. 50 (1985), 535-551

[D1] Damon, J. Deformations of sections of singularities and Gorenstein surface singularities Amer. J. Math. 109 (1987), 695-722

[D2] Higher Multiplicities and Almost Free Divisors and Complete Intersections Memoirs Amer. Math. Soc. 123 no 589 (1996)

[D3] The unfolding and determinacy theorems for subgroups of $\mathcal{A}$ and $\mathcal{K}$ Memoirs Amer. Math. Soc. 50 no. 306 (1984)

[D4] Topological Triviality and Versality for subgroups of $\mathcal{A}$ and $\mathcal{K}$ II: Sufficient Conditions and Applications Nonlinearity 5 (1992), 373-412

[D5] On the Legacy of Free Divisors: Discriminants and Morse-type Singularities Amer. J. Math. 120 (1998), 453-492

[D6] On the Legacy of Free Divisors II: Free* Divisors and Complete Intersections, Moscow Math. Journal 3 no. 2, Special Issue in honor of V. I. Arnol'd, (2003) 361-395

[D7] $\mathcal{A}$-equivalence and the equivalence of sections of images and discriminants Singularity Theory and its Applications: Warwick 1989, Part I Springer Lecture Notes. 1462 (1991), 93-121

[DM] Damon, J. and Mond, D. $\mathcal{A}$-codimension and the vanishing topology of discriminants Invent. Math. 106 (1991), 217-242

[EN] Eagon, J.A. and Northcott, D. Ideals defined by matrices and a certain complex associated with them Proc. Roy. Soc. London 299 (1967), 147-172

[Ga] Galligo, A. Théorème de Division et Stabilité en Géométrie Analytique Locale Annales Inst. Fourier 29 (1979), 107-184

[Go1] Goryunov, V. Projections of generic surfaces with boundaries Adv. Soviet Math. 1 (1990), $157-200$

[Go2] Functions on Space Curves Jour. London Math. Soc. 61 (2000), 807- 822

[K] Kazarian M. E Flattenings of projective curves, singularities of Schubert stratifications of Grassmannian and flag varieties, and bifurcations of Weierstrass points of algebraic curves Russian Math. Surveys 46 (1991), 91-136

[L] Looijenga, E.J.N. Isolated singular points on complete intersections London Math. Soc. Lecture Notes in Math. 77, Cambridge Univ. Press (1984)

[Ly] Lyashko, O. Classification of critical points of functions on a manifold with singular boundary Funct. Anal. and Appl. 17 (1984), 187-193

[Mc] Macaulay, F.S. The algebraic theory of modular systems Cambridge Tracts 19, Cambridge Univ. Press, 1916

[M-IV] Mather, J Stability of $C^{\infty}$-mappings: IV. Classification of stable germs by $\mathbb{R}$-algebras Publ. Math. I.H.E.S. 37 (1969), 223-248

[Mar] Martinet, J. Deploiements versels des applications differentiables et classification des applications stables Singularités d'Applications Differentiables, Plans-sur-Bex, Springer Lecture Notes 535 (1975), 1-44

[Mo] Mond, D. Differential Forms on Free and Almost Free Divisors Proc. London Math. Society 81 (2000) 587-617.

$[\mathrm{MM}]$ Mond, D. and Montaldi, J. Deformations of maps on complete intersections, Damon's $\mathcal{K}_{V^{-}}$ equivalence and bifurcations London Math. Soc. Lect. Notes 201, Cambridge Univ. Press (1994), 263-284

[MVS] Mond, D. and Van Straten, D. Milnor number equals Tyurina number for functions on space curves Jour. London Math. Soc. 63 (2001), 177-187 
[No] Northcott, D. G. Semiregular rings and semi- regular ideals Quart. J. Math. Oxford 11 (1960), 81-104

[Sa] Saito, K. Theory of logarithmic differential forms and logarithmic vector fields J. Fac. Sci. Univ. Tokyo Sect. Math. 27 (1980), 265-291

[Se] Serre, J. P. Algèbra Locale Multiplicités Springer Lecture notes in Math. 11 SpringerVerlag, Berlin., 1965

[Si] Siersma, D. Singularities of functions on boundaries, corners, etc. Quart. J. Math. 32 (1981), 119-127

[Ta] Tari, F. Projections of Piecewise Smooth Surfaces J. London Math. Soc. 44 (1991), 155-172

$[\mathrm{Te}]$ Teissier, B. The hunting of invariants in the geometry of the discriminant in Real and complex singularities, Oslo 1976 (ed. Per Holm), Sijthoof and Noordhoff, Alphen aan den Rijn, 1977, 567-677

[To] Terao, H. The bifurcation set and logarithmic vector fields Math. Ann. 263 (1983), 313-321

[Zl] Zakalyukin, V. Flag Contact Singularities Real and Complex Singularities, eds. J. W. Bruce and F. Tari, Research Notes in Math. 412, Chapman and Hall (2000) 134-146

[Z12] Applications of Flag Contact Singularities, New Developments in Singularity Theory, eds. D. Siersma et al, NATO Science Series Vol 21 Kluwer Academic Publ. (2001) 35-64

Department of Mathematics, University of North Carolina, Chapel Hill, NC 275993250, USA 